\title{
Tanshinone IIA Combined With CsA Inhibit Myocardial Cell Apoptosis Induced by Renal Ischemia-reperfusion Injury by Modulating Mitochondrial Function Through PI3K/Akt/Bad Pathway in Obese Rats
}

He Tai

Liaoning University of Traditional Chinese medicine

Xiao-lin Jiang

Liaoning University of Traditional Chinese medicine

Yue Li

Liaoning University of Traditional Chinese medicine

Liang Kong

Liaoning University of Traditional Chinese medicine

Si-cheng Yao

Liaoning University of Traditional Chinese Medicine

Nan Song

Liaoning University of Traditional chinese medicine

Mei-jun Lv

Liaoning University of Traditional Chinese medicine Jin Wu

Liaoning University of Traditional Chinese medicine

Ping Yang

Liaoning University of Traditional Chinese Medicine

\section{Guan-lin Yang}

Liaoning University of Traditional Chinese medicine

Jin-song Kuang

Fourth Peoplel's Hospital in Shenyang: Shenyang Medical College

Zhi-ming Lan

Shenzhen Traditional Chinese medicine hospital

Lian-qun Jia ( $\sim$ jlq-8@163.com )

Key Laboratory of Ministry of Education for Traditional Chinese Medicine Viscera-State Theory and Applications, Liaoning University of Traditional Chinese Medicine, Shenyang , China https://orcid.org/0000-0003-0923-6987 
Research

Keywords: Renal ischemia-reperfusion, Obesity, Mitochondrial dysfunction, Acute myocardial injury, Tanshinone IIA, Cyclosporine A, Apoptosis, PI3K/Akt/Bad pathway

Posted Date: September 22nd, 2020

DOI: https://doi.org/10.21203/rs.3.rs-77027/v1

License: (c) (i) This work is licensed under a Creative Commons Attribution 4.0 International License. Read Full License 


\section{Abstract}

Background: Acute myocardial injury (AMI), which is induced by renal ischemia-reperfusion (IR), is a significant cause of acute kidney injury (AKI)-related associated death. Obesity increases the severity and frequency of AMI and AKI. Tanshinone IIA (TIIA) combined with cyclosporine A (CsA) pretreatment was used to alleviate myocardial cell apoptosis induced by renal IR, and to determine whether TIIA combined with CsA would attenuate myocardial cell apoptosis by modulating mitochondrial function through the $\mathrm{PI} 3 \mathrm{~K} / \mathrm{Akt} / \mathrm{Bad}$ pathway in obese rats.

Methods: Male rates were fed a high fat diet for 8 weeks to generate obesity. AKI was induced by 30 min of kidney ischemia followed $24 \mathrm{~h}$ of reperfusion. Obese rats were given TIIA (10 mg/kg.d) for 2 weeks and CsA $(5 \mathrm{mg} / \mathrm{kg}) 30 \mathrm{~min}$ before renal IR. Related indicators were examined.

Results: TIIA combined with CsA alleviated the pathohistological injury and apoptosis induced by renal IR in myocardial cells. In addition, TIIA combined with CsA improved cardiac function and decreased the serum myocardial enzyme spectrum in obese rats after renal IR. At the same time, TIIA combined with CsA improved mitochondrial function. Abnormal function of mitochondria was supported by decreases in the respiration controlling rate (RCR), intracellular adenosine triphosphate (ATP), oxygen consumption rate, and mitochondrial membrane potential (MMP), and increases in mitochondrial reactive oxygen species (ROS), opening of the mitochondrial permeability transition pore (mPTP), mitochondrial DNA damage, and mitochondrial respiratory chain complex enzymes $(\mathbb{Q}, \mathbb{Q}, \mathbb{Q}, \mathbb{Q}$, and $\mathbb{\nabla})$. The injury of mitochondrial dynamic function was assessed by a decrease in Drp1, and increases in Mfn1 and Mfn2, and mitochondrial biogenesis injury was assessed by decreases in PGC-1, NRF1, and TFam. TIIA combined with CsA can attenuate apoptosis through modulating mitochondrial function through the $\mathrm{PI} 3 \mathrm{~K} / \mathrm{Akt} / \mathrm{Bad}$ pathway in obese rats.

Conclusion: We used isolated mitochondria from rat myocardial tissues to demonstrate that myocardial mitochondrial dysfunction occurred along with renal IR to induce myocardial cell apoptosis; obesity aggravated apoptosis. TIIA combined with CsA attenuated myocardial cell apoptosis by modulating mitochondrial function through the PI3K/Akt/Bad pathway in obese rats.

\section{Background}

Acute kidney injury (AKI) is a very severe, even life-threatening complication of critically ill patients [1]. Renal ischemia-reperfusion (IR) is the main cause for AKI [2]. Because obesity is closely related to hyperlipidemia, diabetes, and hyperuricemia, which induce insulin resistance and hypertension, they are considered chronic hyper-inflammatory states [3] that enhance the severity and morbidity of renal diseases [4]. Ischemia and AKI rarely occur in the kidneys alone, as they are often combined with multiple organ dysfunction.

Patients with renal insufficiency have a high-risk of triggering cardiovascular diseases, representing $45 \%$ of the cause of death in patients undergoing hemodialysis [5]. The mechanism of acute myocardial injury 
(AMI) induced by renal IR is indeterminate, including kidney dysfunction which results in a hyperinflammatory state and volume overload [6]. Renal IR can lead to impactful changes in heart morphology combined with increased microvasculature [7]. The study has demonstrated that the IR mechanism is closely related with mitochondrial dysfunction in heart [8]. However, no study has explored mitochondrial dysfunction in myocardial cells induced by renal IR.

Reducing the inflammatory response and resisting oxidant stress are the main biological activities of tanshinone IIA (TIIA), the main active ingredient in Salvia miltiorrhiza Bge [9]. In addition, TIIA plays a protective role in myocardial ischemia [10]. The study has demonstrated that TIIA represses opening of the mitochondrial permeability transition pore (mPTP), which is cardioprotective [11]. Cyclosporine A (CsA) inhibits opening of the MPTP by binding to cyclophilin D (CyP-D) to minimize IR damage [12]. Injecting CsA before ischemia protects renal function [13]. However, few studies have investigated the myocardial protective properties of TIIA and CsA by repressing opening of the MPTP.

Cell survival is associated with the phosphoinositide-3 kinase (PI3K) pathway, which relies on Akt kinase phosphorylation and activation followed by the proapoptotic Blc-2 family protein Bad phosphorylation and inhibition. PI3K plays an important role in growth factor signal transduction. Under various cytokines and the activation of physiochemical factors, PI3K generates myoinositol as a second messenger. Akt also plays crucial roles in many biological processes, such as the cell cycle, cell metabolism, apoptosis, and cell growth [14]. Mitochondrial-mediated apoptosis is inhibited through the PI3K/Akt/Bad signaling pathway [15]. However, few studies have investigated the myocardial protective roles of TIIA and CsA by modulating mitochondrial function through the PI3K/Akt/Bad pathway.

The current research focused on assessing an AKI method (induced by IR) leading to dysfunction of myocardial mitochondria, using a combination of TIIA and CsA to identify new therapeutic targets.

\section{Materials And Methods}

\section{Experimental animals and Ethical statement}

We used male Sprague-Dawley rats (weight $180-220 \mathrm{~g}$ and 8 weeks old) in our experiments. The animals were acquired from Liaoning Changsheng Biotechnology Co., Ltd. (Production License: SCXK (Liao) 2015-0001). The rats were kept in cages under controlled conditions of $20 \pm 3^{\circ} \mathrm{C}$ and $45-65 \%$ humidity, and with a $12 \mathrm{~h}$ light/dark cycle (lights on at 06:00 h), and fed water and a pelleted diet ad libitum. Animal experimental design and husbandry procedures were approved by the Ethical Committee of Animal Handling (2019019) at Liaoning University of Traditional Chinese Medicine and complied with the guidelines of the Use and Care of laboratory animals published by US National Institutes of Health. We did our best to decrease the number of rats and suffering during the study.

\section{Chemicals and Reagents}


TIIA (Sulfotanshinone Sodium Injection, $10 \mathrm{mg}$ each) was produced at the Shanghai NO. 1 Biochemical Pharmaceutical Co., Ltd. (Shanghai, China). TIIA was dissolved in deionized water to obtain a stock solution ( $5 \mathrm{mg} / \mathrm{ml})$. CsA (20 mg each) was produced at Beijing Solarbio Science \& Technology Co., Ltd. (Beijing, China) and was dissolved in $0.1 \%$ dimethylsulfoxide to a stock solution $(2.5 \mathrm{mg} / \mathrm{ml})$, which was further diluted to achieve the ideal concentration. All of the above stock solutions were used in a timely manner.

\section{Animal Grouping and Methods of drug dosing}

A total of 120 rats were randomly divided into six groups, including the sham operation group, the IR group, the IR (obese) group, the TIIA group, the CsA group, and the TIIA+ CsA group. Each group included 20 rats. All rats were fed a general maintenance feed for 2 weeks to adapt to the environment. Then, the sham and IR groups continued to eat the general maintenance feed for 8 weeks, whereas the other four groups were fed a high-fat diet (HFD) for 8 weeks. Rats in the sham group, the IR, and IR (obese) groups were given deionized water. The TIIA group was given an intraperitoneal injection of TIIA $(10 \mathrm{mg} / \mathrm{kg} \cdot \mathrm{d})$ for 2 weeks before renal IR; the CsA group was given an intraperitoneal injection of CsA ( $5 \mathrm{mg} / \mathrm{kg}) 30 \mathrm{~min}$ before renal IR; and the TIIA + CsA group was given an intraperitoneal injection of TIIA $(10 \mathrm{mg} / \mathrm{kg} \cdot \mathrm{d})$ for 2 weeks before renal IR+ intraperitoneal injection CsA ( $5 \mathrm{mg} / \mathrm{kg}) 30 \mathrm{~min}$ before renal IR. The components of the HFD included $25 \%$ total fat, $18 \%$ protein, $44 \%$ carbohydrate, $13 \%$ fiber, and $11 \%$ unsaturated fat, ash, and other ingredients [16]. Rats that gained $30 \%$ of their body weight were chosen for further study [17].

\section{Surgical procedure}

The rats were anaesthetized with thiopental sodium $(120 \mathrm{mg} / \mathrm{kg})$ by intraperitoneal injection, and pinching of the tail and paw was used to evaluate the anesthetic effect. The abdomen was opened to expose the right kidney, and the renal pedicle was separated to expose the renal vessels. A 3-0 silk suture was used to ligate the renal vessel, and the right kidney was removed. The left kidney was exposed and the renal vessels were separated. An arterial clamp was used to clamp off the left renal artery for 30 min to build ischemia. After $30 \mathrm{~min}$ of ischemia, the arterial clamp was removed for $24 \mathrm{~h}$ of reperfusion. The left kidney was observed for 15 min to ensure normal blood reperfusion, which was shown by a red color [18]. The incision was closed with 3-0 silk sutures. The rats were placed on a heating pad to maintain $37^{\circ} \mathrm{C}$ body temperature throughout the experimental procedure. Sham-operated rats received the same surgical procedure without using an arterial clamp to clamp off the left renal artery [19].

\section{Serum myocardial enzyme spectrum and inflammatory factor analyses}

Arterial blood samples were used for the myocardial enzyme spectrum analysis. A $0.5 \mathrm{~mL}$ aliquot of arterial blood was drawn from the abdominal aorta (at the end of the reperfusion period), and creatine kinase isoenzymes-muscle/brain (CK-MB), cardiac troponin I (cTNI), tumor necrosis factor-a (TNF-a), and interleukin-1 $\beta$ (IL-1 $\beta$ ) were measured with kits (KHB, Shanghai, China).

\section{Cardiac function analysis}


Noninvasive transthoracic echocardiography (vevo2100; Visualsonics, Toronto, ONT, Canada) was used to evaluate the function and morphology of the left ventricle in anesthetized animals (Matrx VIP 3000) (Figure 1A). This method consisted of two-dimensional mode; that is, time-motion mode and blood flow measurements in pulsed Doppler mode. Left ventricular end-systolic internal diameter (LVIDs) and left ventricular end-diastolic internal diameter (LVIDd), as well as the left ventricular ejection fraction (EF) and left ventricular fractional shortening (FS) were recorded [20].

\section{Histological assessment of the myocardium by hematoxylin and eosin (HE) staining}

Myocardial tissues were cut into 5- $\mu \mathrm{m}$ thick sections and stained with $\mathrm{HE}$ after paraffin embedding as described previously [21]. We used paraformaldehyde (4\%) to immerse the myocardial tissues for $24 \mathrm{~h}$ and then transferred the tissues to ethanol (70\%). The myocardial tissues were observed under light microscopy [22]. The degree of injury in myocardial tissues was scored on a scale from 0 to 3 [23]: 0 , normal myocardial tissues; 1 (mild), interstitial edema and localized necrosis; 2 (moderate), widespread myocardial cell swelling and necrosis; 3 (severe), necrosis with contraction bands, neutrophil infiltration, and compressed capillaries; and 4 (highly severe), diffuse necrosis with contraction bands, neutrophil infiltration, and compressed capillaries and hemorrhaging.

\section{Electron microscopy to observe mitochondria}

Myocardial tissues were obtained immediately after anesthesia and cut into small pieces $\left(1 \mathrm{~mm}^{3}\right)$. The specimens were fixed in glutaraldehyde $(2 \%)$ at $4{ }^{\circ} \mathrm{C}$, washed with $\mathrm{pH} 7.4 \mathrm{PBS}(0.1 \mathrm{~mol} / \mathrm{L})$, fixed with osmium tetroxide (1\%), and stained with aqueous uranyl acetate (1\%). Using capsules included embedding medium to place the s pecimens $70^{\circ} \mathrm{C}$ for about $48 \mathrm{~h}$. The sections were stained with uranyl acetate and alkaline lead citrate for observation with an electron microscope (H-7650; Hitachi, Tokyo, Japan).

\section{Apoptosis assessment of the myocardial tissue using TUNEL}

The terminal deoxynucleotidyl transferase-mediated dUTP nick end-labeling (TUNEL) assay was used to detect apoptosis with an In Situ Cell Death Detection Kit, POD (Roche, Mannheim, Germany). We used 5$\mu \mathrm{m}$ thick sections for TUNEL staining [21]. The sections were incubated for $15 \mathrm{~min}$ in Protease K (10 $\mu \mathrm{g} / \mathrm{mL}$ ) after deparaffinization and rehydration. A fresh TUNEL reaction mixture was added to the samples, which were incubated $\left(37^{\circ} \mathrm{C}\right)$ for $60 \mathrm{~min}$ in the dark. After washing, $0.1 \mu \mathrm{g} / \mathrm{mL}$ 4',6-diamidino-2phenylindole (DAPI) (Beyotime) was used to stain the cell nuclei. The samples were analyzed in a drop of phosphate buffered saline (PBS) under a fluorescence microscope (Canon, Tokyo, Japan). We observed eight random visual fields per animal to determine the number of TUNEL-positive cells in a high-power field.

\section{Caspase-3/9 activity}


Caspase-3/9 activity were detected in myocardial tissues by the fluorescent caspase-specific substrate AcDEVD-7-pNA (Solarbio). A $10 \mathrm{mg}$ portion of myocardial proteins was added to reaction buffer and incubated at $37^{\circ} \mathrm{C}$ for $2 \mathrm{~h}$. Enzyme-catalyzed release was quantified by a fluorimeter at $405 \mathrm{~nm}$.

\section{Preparation of the mitochondrial suspension}

As previously reported in liver [24], the animals were sacrificed by cervical dislocation, and the heart was quickly removed and placed in an ice-cold pH 7.4 buffer (1 mM EDTA, $250 \mathrm{mM}$ sucrose, and $10 \mathrm{mM}$ Tris$\mathrm{HCl})$. After trimming, the myocardial tissues $(50-100 \mathrm{mg}$ ) were homogenized in an isolation buffer. The entire isolation process was performed at $4^{\circ} \mathrm{C}$. The supernatant was collected after centrifugation in 700 $\times \mathrm{g}$ for $10 \mathrm{~min}$, and the supernatant was centrifuged again at 7,000 $\times \mathrm{g}$ for $10 \mathrm{~min}$. The resulting supernatant was discarded, and the mitochondrial pellet was resuspended in $5 \mathrm{ml}$ isolated buffer followed by centrifuging twice at 7,000 $\times \mathrm{g}$ for $10 \mathrm{~min}$. A clean mitochondrial solution was acquired and preserved in mitochondrial preservation solution (20 mM sucrose, $10 \mathrm{mM} \mathrm{KH}_{2} \mathrm{PO}_{4}, 2 \mathrm{mM} \mathrm{MgCl}_{2}, 100 \mathrm{mM}$ $\mathrm{KCl}, 5 \mathrm{mM} \mathrm{HEPES}$, and $1 \mathrm{mM}$ EDTA) to generate a $5 \mathrm{mg} / \mathrm{mL}$ mitochondrial protein suspension, which was placed on ice for immediate use. The bicinchoninic acid (BCA) reagent box (Beyotime) was used to measure the protein concentration of the mitochondrial suspension $(100-1000 \mu \mathrm{g} / \mathrm{ml})$. The mitochondrial suspension was used to measure the mitochondrial membrane potential (MMP), ATP synthesis, opening of the mitochondrial permeability transition pore (MPTP), reactive oxygen species (ROS), and the respiratory control rate $(\mathrm{RCR})$.

\section{Measurement of the MMP}

A mitochondrial membrane potential assay kit (Beyotime) was used to detect the MMP. The prepared JC1 working solution was diluted five times with JC-1 Dyeing Buffer (1X); a $0.9 \mathrm{ml}$ aliquot of the diluted JC-1 working solution was added to $0.1 \mathrm{ml}$ of the purified mitochondria (protein content 100-1000 $\mu \mathrm{g} / \mathrm{ml}$ ). We used a fluoroenzyme label to detect MMP; $1 \mathrm{ml}$ of the mixture was added to the fluoroenzyme-labeled assay with the emission wavelength set to $530 \mathrm{~nm}$ and excitation wavelength set to $490 \mathrm{~nm}$, and the $\mathrm{red} / \mathrm{green}$ value was calculated.

\section{Opening of the MPTP}

Opening of the mPTP was measured by detecting absorbance at $540 \mathrm{~nm}$ of mitochondria exposed to 250 $\mu \mathrm{M} \mathrm{CaCl}_{2}$; "-": treatment without $\mathrm{Ca}^{+}$; "+": treatment with $\mathrm{Ca}^{+}$. The Purified Mitochondrial Membrane Pore Channel Colorimetric Assay kit (GENMED, Shanghai, China) was used according to the manufacturer's protocol. $\mathrm{CaCl}_{2}(200 \mu \mathrm{mol} / \mathrm{L})$ was used to induce opening of the mPTP. The optical density (OD) value from 0 to $10 \mathrm{~min}$ at $520 \mathrm{~nm}$ was read from an ultra-micro microporous plate spectrophotometer (Biotek, Winooski, VT, USA). A decrease in the OD value reflects opening of the MPTP. The OD value recorded at the onset of the experiment ( $0 \mathrm{~min}$ ) represents minimum optical density ( $\mathrm{min} O D)$; the OD value recorded at the end of the experiment (10 $\mathrm{min}$ ) represents maximum optical density ( $\mathrm{min} \mathrm{OD}$ ). Min/max OD is negatively associated with the extent of MPTP opening [24, 25]. 


\section{Measurement of ROS}

ROS were detected using the multi-mode microplate reader and the $2^{\prime}, 7^{\prime}$-dichlorodihydrofluorescein diacetate fluorescent probe (DCFH-DA).

\section{Measurement of oxygen consumption rate and RCR}

Mitochondrial oxygen consumption rate was detected using a Clark-type electrode connected to a thermostatic water bath and calibrated with using air-saturated medium (contain $432 \mathrm{nmol} 0 / \mathrm{ml}$ at 32 $\left.{ }^{\circ} \mathrm{C}\right)$ to calibrat.

RCR (ratio of state III to state IV) is an index used to assess oxidative phosphorylation, respiratory chain function, and integrity of isolated mitochondria. The RCR was evaluated using an Oxytherm Clark-type oxygen electrode (OXYT1/ED; Hansatech Instruments, Norfolk, UK). Mitochondria (60 $\mu \mathrm{g}$ isolated from myocardiu) were placed in the oxytherm chamber containing respiration buffer (20 mM HEPES, $125 \mathrm{mM}$ $\mathrm{KCl}, 0.1 \% \mathrm{BSA}, 2.5 \mathrm{mM} \mathrm{KH}_{2} \mathrm{PO}_{4}$, and $2 \mathrm{mM} \mathrm{MgCl}_{2}, \mathrm{pH} 7.2$ ), and stirred at $37^{\circ} \mathrm{C}$. The slope of the response of mitochondria to consecutive administrations of respiration substrates was defined as the rate of oxygen consumption for each respiratory state, as reported previously [26].

\section{Measurement of damaged mtDNA}

Damaged mitochondrial DNA (mtDNA) was assessed through the ratio of long and short fragments using the real-time quantitative polymerase chain reaction (RT-qPCR). DNA was isolated by extracting total DNA using the Genomic-tip 20/G kit (Qiagen, Valencia, CA, USA) according to the manufacturer's protocol. The PCR products and purified DNA were quantified fluorometrically using the Picogreen ds DNA reagent (Invitrogen, Milan, Italy). RT-qPCR was performed on the DNA extracts as reported previously using the following modifications [27]. PCR amplification was conducted using the Ranger DNA Polymerase with appropriate premixes (Bioline Ltd., London, UK). The two primer pairs (mtDNA long fragment and short fragment) (General Biosystems, Anhui, China) are shown in Table 1. The mtDNA long fragment was amplified using the standard thermocycler program, including initial denaturation at $94^{\circ} \mathrm{C}(1 \mathrm{~min}), 94{ }^{\circ} \mathrm{C}$ (15 s) for 18 cycles, $65^{\circ} \mathrm{C}(12 \mathrm{~min})$, and a final extension at $72{ }^{\circ} \mathrm{C}(10 \mathrm{~min})$. The short mtDNA fragment was amplified using the same program except the extension temperature was $60^{\circ} \mathrm{C}$.

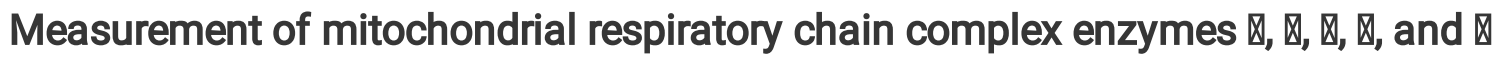

The myocardial tissues $(100 \mathrm{mg})$ were homogenized in an isolation buffer. The entire isolation process

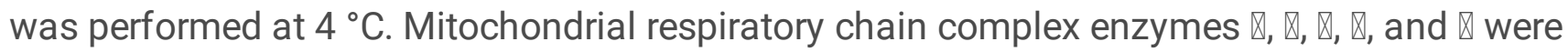
independently assessed using the independent Assay Kit (Solarbio). Enzyme-catalyzed release were quantified by a fluorimeter at $340,605,550,550$, and $660 \mathrm{~nm}$ separately.

\section{Measurement of ATP}


The concentration of ATP in isolated mitochondria was detected using a luciferase-based luminescence enhanced ATP assay kit (Beyotime). Isolated mitochondria ( $1 \mathrm{mg} / \mathrm{mL}$ protein) were incubated in respiration buffer $(0.5 \mathrm{~mL})$ with $2.5 \mathrm{mM}$ malic acid, $2.5 \mathrm{mM}$ succinate, and $2.5 \mathrm{mM}$ ADP for $10 \mathrm{~min}$. The ATP concentrations in the mitochondrial suspension and cell lysates were determined using a SpectraMax Paradigm Multi-Mode Microplate Reader (Molecular Devices, Sacramento, CA, USA).

\section{RNA extraction and cDNA synthesis}

Trizol reagent (Invitrogen, Carlsbad, CA, USA) was used to isolate total genomic RNA, and the quality of isolated RNA was determined by spectrophotometry $(260 \mathrm{~nm})$. Reverse transcription was performed with total RNA $(1 \mu \mathrm{g})$ and the M-MLV Reverse Transcriptase Kit (Promega A3500; Promega, Madison, WI, USA). Briefly, the total reaction volume $(40 \mu \mathrm{L})$ was used in a Veriti 96 Well Thermal Cycler long PCR system (Applied Biosystems, Foster City, CA, USA) with the following reaction conditions: $72{ }^{\circ} \mathrm{C}$ for $3 \mathrm{~min}, 42{ }^{\circ} \mathrm{C}$ for $90 \mathrm{~min}, 70^{\circ} \mathrm{C}$ for $15 \mathrm{~min}$, and hold at $4{ }^{\circ} \mathrm{C}$.

\section{Real-time qPCR}

RT-qPCR was used to detect the copy number of specific genes at the transcriptional level using a cDNA template. PCR was performed on a Rotor-Gene Q Sequence Detection System (Qiagen, Hilden, Germany) using SYBR Premix Ex Taqll (Takara Bio, Shiga, Japan) [28]. PCR was performed in a $20 \mu \mathrm{L}$ system (0.5 $\mu \mathrm{M}$ primers $+1 \mu \mathrm{L}$ synthetic cDNA $+10 \mu \mathrm{L}$ SYBR Premix Ex Taq II) under the following conditions: $95^{\circ} \mathrm{C}$ for $10 \mathrm{~min} ; 95^{\circ} \mathrm{C}$ for $10 \mathrm{~s}, 40$ cycles, $60^{\circ} \mathrm{C}$ for $15 \mathrm{~s} ; 72^{\circ} \mathrm{C}$ for $20 \mathrm{~s} ; 72^{\circ} \mathrm{C}$ for $10 \mathrm{~min}$. The value was calculated with GAPDH as the internal control [29]. Sequences of two pairs of PCR primers were used and are shown in Table 1.

\section{Protein detection}

Total proteins were extracted from tissues using RIPA Lysis Buffer. Protein concentration was measured using the BCA Protein Assay Kit. An equal amount of total protein was subjected to 8-12\% sodium dodecyl sulfate-polyacrylamide gel electrophoresis, and the proteins were transferred to a PVDF membrane. After blocking in skim milk solution, the membrane was incubated overnight separately with anti-B-actin, anti-PI3K, anti-p-Akt, anti-Akt, anti-p-Bad, anti-Bad, anti-Bcl-2, anti-Bax, anti-Cyt-c, anti-caspase9, anti-cleaved caspase-9, anti-caspase-3, anti-cleaved caspase-3, anti-PARP, anti-Drp1, anti-Mfn1, antiMfn2, anti-PGC-1, anti-NRF1, and anti-TFam antibodies (antibodies shown in Table 2). Then, the membrane was incubated with secondary HRP-conjugated goat anti-rabbit antibodies (Santa Cruz Biotechnology, Santa Cruz, CA, USA). The proteins were visualized using an enhanced chemiluminescence kit from Thermo Fisher Scientific (Waltham, MA, USA). Alpha View software (Cell Biosciences, Preston VIC, Australia) was used to perform the densitometric analysis.

\section{Statistical analysis}

The SPSS statistical package (version 17.0, SPSS Inc. Chicago, IL, USA) was used for the statistical analysis. Data are expressed as mean \pm standard error. One-way analysis of variance (ANOVA) was used 
to compare the six independent groups and the $2 \times 2$ comparisons between the groups. The LSD- $t$ test was used to compare among the six groups. A $P$-value $<0.05$ was considered significant.

\section{Results}

\section{The cause of death during the experiment}

Eventually, 66 rats (13 (65.00\%) in the sham group, 13 (65.00\%) in the IR group, 10 (50.00\%) in the IR (obese) group, 10 (50.00\%) in the TIIA group, 11 (55.00\%) in the CsA group, and 9 (45.00\%) in the TIIA+CsA group) completed this study; 54 rats died and the reasons are described in Table 3 .

\section{TIIA combined with CsA improves cardiac function, myocardial enzymes, and inflammatory factors induced by renal IR in obese rats}

We assessed cardiac function (Figure 1B), myocardial enzymes (Figure 1C), and inflammatory factors (Figure 1D) to further assess myocardial injury and the protective effect of TIIA+CsA.

The cardiac function (EF, FS) values decreased in the IR and IR (obese) groups, particularly in the IR (obese) group, compared with the sham group $(p<0.05)$. Cardiac function (LVIDs, LVIDd) values increased in the IR and IR (obese) groups, particularly in the IR (obese) group, compared with the sham group $(p<0.05)$, which was improved by pretreatment with TIIA, CsA, and TIIA+CsA (Figure. 1B). The CK$\mathrm{MB}$ and cTNI values increased in the IR and IR (obese) groups, particularly in the IR (obese) group, compared with the sham group $(p<0.05)$, which was improved by pretreatment with TIIA, CsA, and TIIA+CsA (Figure 1C). Serum inflammatory factor (TNF-a and IL-1 $\beta$ ) values increased in the IR and IR (obese) groups, particularly in the IR (obese) group, compared with the sham group $(p<0.05)$, which decreased after the pretreatment with TIIA, CsA, and TIIA+CsA (Figure 1D).

\section{TIIA combined with CsA improves the pathological structure of the myocardium induced by renal IR in obese rats}

We investigated the protective effect of TIIA combined with CsA on myocardial injury after IR in obese rats by observing HE-stained myocardial tissue (Figure. 2A). The myocardial cells in the sham and TIIA+ CsA groups were similar and only had slight interstitial edema and localized necrosis. Many disordered myocardial cells combined with interstitial edema were detected in the IR and IR (obese) groups. However, pretreatment with TIIA, CsA, and TIIA+CsA alleviated the myocardial injury, as shown by improvements in interstitial edema, and decreased disorder of the myocardial cells. All injury changes were assessed by a histological score (Figure. 2A). The statistical results showed that the myocardial injury score in the IR and IR (obese) groups increased compared with that in the sham group $(p<0.05)$; the score in the IR (obese) group was higher than that in the IR group $(p<0.05)$. All injury scores decreased in the TIIA, CsA, and TIIA+CsA groups $(p<0.05)$ (Figure 2A).

Myocardial mitochondrial morphological changes induced by renal IR in obese rats 
We evaluated mitochondrial function to further assess myocardial injury and the protective effect of TIIA+CsA. We used an electron microscope to observe the changes in mitochondrial morphology (Figure 2B).

The electron microscopic pictures $(40,000 \times)$ of the myocardial tissues revealed that myocardial cells in the IR and IR (obese) groups exhibited abnormal mitochondrial morphology with swelling or membrane rupture (paired white arrows) following renal IR. The sham group exhibited normal mitochondrial morphology (single white arrow). The number of abnormal mitochondria increased in the IR and IR (obese) groups, particularly in the IR (obese) group, compared with the sham group, TIIA, CsA, and TIIA+CsA groups. Pretreatment with TIIA, CsA, or TIIA+CsA improved the changes in mitochondrial morphology (Figure 2B). Percentage of damaged mitochondria in myocardial tissue from the IR and IR (obese) groups increased compared with those in the sham group $(p<0.05)$. However, pretreatment with TIIA, CsA, or TIIA+CsA decreased percentage of damaged mitochondria (Figure 2B).

\section{TIIA combined with CsA reduces myocardial cell apoptosis induced by renal IR in obese rats}

The TUNEL assay was used to investigate the protective effect of TIIA combined with CsA on myocardial cell apoptosis induced by IR in obese rats (Figure $3 \mathrm{~A}$ ). The numbers of apoptotic cells in myocardial tissue from the IR and IR (obese) groups increased compared with those in the sham group $(p<0.05)$. However, pretreatment with TIIA, CsA, or TIIA+CsA alleviated myocardial cell apoptosis (Figure 3A). Caspase-9 and caspase-3 were significantly activated in the IR and IR (obese) groups than the sham group $(p<0.05)$. However, pretreatment with TIIA, CsA, or TIIA+CsA decreased caspase-9/3 activity in myocardial cells (Figure 3B, C). We used western blot to detect the cleaved caspase-9/3 (Figure 3D), the cleaved caspase- $9 / 3$ in myocardial tissue from the IR and IR (obese) groups increased compared with those in the sham group $(p<0.05)$. However, pretreatment with TIIA, CsA, or TIIA+CsA can decrease the cleaved caspase-9/3 $(p<0.05)$ (Figure 3D).

\section{TIIA combined with CsA improves mitochondrial function induced by renal IR in obese rats}

We detected the MMP (ratio of red/green), opening of the MPAP (\%), mitochondrial ROS, mIDNA, oxygen

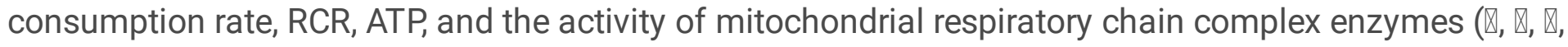

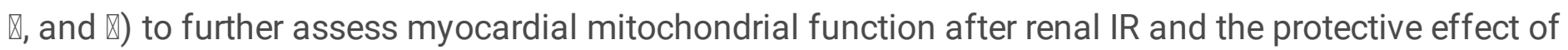
TIIA+CsA. Mitochondria were isolated from rat myocardial tissues. Renal IR increased the mitochondrial ROS level and opening of the MPAP (\%), particularly in the IR (obese) group, compared with the sham group $(p<0.05)$. The mitochondrial ROS level was alleviated by pretreatment with TIIA, CsA, or TIIA+CsA $(p<0.05)$. Renal IR decreased the mitochondrial oxygen consumption rate, RCR, and the MMP (ratio of $\mathrm{red} /$ green), particularly in the IR (obese) group, compared with the sham group $(p<0.05)$. Pretreatment with TIIA, CsA, and TIIA+CsA significantly increased these factors $(p<0.05)$. Real-time qPCR was used to measure the levels of mtDNA oxidative damage. The ratio of long/short fragments decreased in the IR and IR (obese) groups, particularly in the IR (obese) group, compared with the sham, TIIA, CsA, and TIIA+CsA groups $(p<0.05)$. Pretreatment with TIIA, CsA, and TIIA+CsA increased the ratio of long/short mtDNA fragments $(p<0.05)$ (Figure 4A). Renal IR decreased the mitochondrial respiratory chain complex 


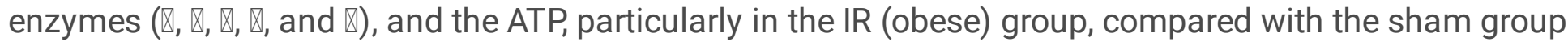
$(p<0.05)$. Pretreatment with TIIA, CsA, and TIIA+CsA significantly increased these enzymes $(p<0.05)$ (Figure 4B).

\section{TIIA combined with CsA improves mitochondrial biogenesis and the dynamics induced by renal IR in obese rats}

We used real-time qPCR and western blot to detect mitochondrial biogenesis and dynamics. We chose PPARy coactivator-1-a (PGC-1a), nucleo respiratory factor1 (Nrf1), and transcription factor A of mitochondrial (Tfam) to represent mitochondrial biogenesis, and dynamin-related protein 1 (Drp1; fission) and mitofusins (Mfns; Mfn1 [fusion], Mfn2 [fusion]), and Drp1 to represent mitochondrial dynamics (fusion and fission processes). Our results show that PGC-1a, Nrf1, Tfam, and Drp1 mRNA levels decreased in the IR and IR (obese) groups, particularly in the IR (obese) group, compared with the sham group $(p<0.05)$. Pretreatment with TIIA, CsA, and TIIA+CsA increased these mRNA levels $(p<0.05)$. Mfn1 and Mfn2 mRNA expression increased in the IR and IR (obese) groups, particularly in the IR (obese) group, compared with the sham group $(p<0.05)$. The mRNA levels of these factors decreased after pretreatment with TIIA, CsA, and TIIA+CsA $(p<0.05)$ (Figure $5 \mathrm{~A})$. The western blot of the biogenesis and dynamics factors showed consistent results (Figure 5B).

\section{TIIA combined with CsA modulates the PI3K/Akt/Bad pathway}

We used real-time qPCR and western blot to detect the target proteins in the PI3K/Akt/Bad pathway in mRNA and protein levels. Renal IR increased the mRNA level expression of Bax, Cyt-c, caspase-9, caspase-3, and PARP significantly, particularly in the IR (obese) group, compared with the sham group ( $p$ $<0.05)$. The mRNA level expression of these factors decreased after pretreatment with TIIA, CsA, and TIIA+CsA $(p<0.05)$. Renal IR decreased the mRNA level expression of PI3K, Bad, Akt, and Bcl-2 significantly, particularly in the IR (obese) group, compared with the sham group $(p<0.05)$. The mRNA level expression of these factors increased after pretreatment with TIIA, CsA, and TIIA+CsA $(p<0.05)$ (Figure 6A). The western blot of the biogenesis and dynamics factors showed consistent results (Figure 6B). TIIA, CsA, and TIIA+CsA induced Akt and Bad phosphorylation (enhanced p-Bad/Bad and p-Akt/Akt) (Figure. 6B).

\section{Discussion}

The main cause of AKI in critically patients is renal ischemia-reperfusion injury (IRI). Continuous renal replacement therapy is the main AKI treatment for critically ill patients, however, it is not sufficient to reduce mortality, multiple organ dysfunction syndrome, and interorgan crosstalk induced by extrarenal complications, which are causes of death from AKI [30]. Renal IRI increases the inflammatory response and cytokine levels, which damage remote organs, such as the heart and lungs [30]. Hyperlipidemia is a risk factor for cardiovascular disease, and it can increase damage by increasing inflammation and ROS [31]. In our study, we prepared the obesity model by feeding the HFD. Renal IRI stimulates a series of complex changes in cells that induce necrosis and apoptosis in cells. Oxidative stress increases 
inflammation and ROS induce the release of pro-inflammatory mediators during the reperfusion phase. These factors play an important role in the pathophysiological course of renal IR. Several antioxidants and anti-inflammatory agents play effective roles reducing the degree of injury. These antioxidants increase the survivability of the kidney to IR injury [32].

AKI often combines with AMI in critically ill patients. Despite that there is a definite relationship between the kidney and the heart, the specific mechanism of the pathophysiological change between the kidney and the heart is unknown. This phenomenon has become controversial because of the confusing results. Despite patient use of dialysis, mortality (50\%) is still very high [33], so a new method must be identified to decrease mortality from AKI. In our study, we prepared the IRI renal model in obese rats and discovered that renal IRI decreased cardiac function, particularly in obese rats, and that TIIA, CsA, and TIIA+CsA improved cardiac function (Figure 1B). Additionally, renal IRI increased myocardial enzyme (CK-MB and cTNI) levels, particularly in obese rats. In contrast, TIIA, CsA, and TIIA+CsA improved cardiac function (Figure 1C). Taken together, renal IRI induced myocardial injury (decreased cardiac function and increased myocardial enzymes), whereas TIIA, CsA, and TIIA+CsA improved myocardial injury.

Renal IRI is involved in the adaptive and innate immune responses and releases cytokines in the kidney [34]. Once this process starts, soluble mediators and remote organs, such as the lung and heart, become damaged through organ crosstalk. The kidney-heart interaction includes several inflammatory mediators in the pathophysiological process of cardiorenal syndrome [35], and the elevated levels of inflammatory cytokines adversely affect myocardial function.

Increased levels of TNF- $a$ and IL- 6 are related with the development of congestive heart failure and mortality in patients with congestive heart failure [36]. Several pathways, such as activation of inflammatory transcription factors and the induction of inflammatory cytokines and genes, contribute to heart damage following renal IRI. In our study, renal IRI induced AMI. We used enzyme-linked immunosorbent assays to detect TNF- $\alpha$ and IL-1 $\beta$, and our results show that renal IRI increased serum levels of TNF- $\alpha$ and IL-1 $\beta$, particularly in the IR (obese) group, compared with the sham group $(p<0.05)$, which decreased after the pretreatment with TIIA, CsA, and TIIA+CsA (Figure 1D). Systemic inflammation regulates cardiac tissue structure, as distinct sections of heart tissues have different collagen content [37].

HE staining of rat myocardial tissue revealed that renal IRI induced a disordered structure, edema, and localized necrosis in myocardial cells, particularly in the IR (obese) group, combined with interstitial edema. However, pretreatment with TIIA, CsA, and TIIA+CsA alleviated the myocardial injury, as shown by improvements in interstitial edema and decreases in the number of disordered myocardial cells (Figure 2A). Structural damage to myocardial tissues also decreased cardiac function (Figure 1B). The statistical results showed that the myocardial injury score in the IR and IR (obese) groups increased compared with that in the sham group $(p<0.05)$. The score in the IR (obese) group was higher than that in the IR group $(p<0.05)$. Pretreatment with TIIA, CsA or TIIA+CsA, decreased the injury scores $(p<0.05)$ (Figure $2 A)$. At the same time, the TUNEL assay showed that renal IRI induced myocardial cell apoptosis (Figure $3 A$ ), 
whereas TIIA+CsA decreased the number of apoptotic cells (Figure 3A). Caspases are major executors of apoptosis related to the final stages of apoptosis. Activation of effector caspase- $9 / 3$ is an important indicator of apoptosis, so caspase-9/3 activity was measured in the present study. Caspase- $9 / 3$ was significantly activated in the IR and IR (obese) groups compared to the sham group, particularly in the IR (obese) group $(p<0.05)$. However, TIIA, CsA, or TIIA+CsA decreased caspase-9/3 activity in myocardial cells (Figure 3B, C). These results are similar to those of Juanjuan Liet al [38]. in an acute lung injury model induced by renal IRI. As the active form of caspase-3, cleaved caspase-3 can induce apoptosis, IR can increase the cleaved caspase-9/3, However, pretreatment with TIIA, CsA, or TIIA+CsA can decrease the cleaved caspase-9/3 $(p<0.05)$ (Figure 3D).

According to previous studies, some anti-inflammatory agents have been discovered, including amelanocyte stimulating hormone, anti-apoptotic agents, and IL-6 inhibitors [38, 39]. A previous study reported that renal IR induces dysfunction in lung mitochondria and dexmedetomidine attenuates lung inflammation, apoptosis, and the MMP [38]. However, until now, no study has investigated myocardial mitochondrial dysfunction induced by renal IR, and no anti-mitochondrial dysfunction agents have been discovered to protect against AMI induced by renal IR in obese rats. Using only the MMP to evaluate the mitochondrial function is too circumscribed, so more methods must be used to evaluate mitochondrial function. We chose the MMP, mitochondrial ATP, opening of the MPTP, mitochondrial ROS, mTDNA, and mitochondrial biogenesis/dynamics to comprehensively evaluate mitochondrial function.

As a universal medicinal herb, it has been demonstrated that S. miltiorrhiza Bge eliminates toxic substances from the blood, accelerates fibrinolytic enzyme activity and thrombolysis, reduced blood viscosity, and protects the cardiovascular system and blood vessels [39]. TIIA is the main active ingredient in S. miltiorrhiza Bge. Pretreatment with TIIA relieves renal injury induced by IR by downregulating the expression of myeloperoxidase (MPO), caspase-3, and inflammation [39]. TIIA decreases the generation of ROS and release of Cyt-c, reduces the frequency of apoptosis, inactivates caspase-3, and inhibits opening of the MPTP [40]. Because CsA inhibited opening of the MPTP, it has been implicated as a treatment target at the beginning of reperfusion [40]. It is now widely believed that mitochondrial dysfunction (particularly opening of the MPTP) plays an important role aggravating injury following renal IRI [41]. Opening of the MPTP decreased the membrane potential and mitochondria swelling, which inhibited oxidative phosphorylation. Opening of the MPTP occurs through binding of the CyP-D protein to the mitochondrial inner membrane. Previous studies have shown that CsA protects against IRI through CyP binding, independently of the anti-calcineurin properties; thus, inhibiting opening of the MPTP [42]. However, no study has explored whether CsA inhibits opening of the mPTP to inhibit the myocardial mitochondrial dysfunction induced by renal IRI. Mitochondrial function is the pathophysiological bridge between kidney-heart interactions during renal IRI. We hypothesized that TIIA combined with CsA would play a protective role in ALI by improving mitochondrial function.

Mitochondria are the energy center of the cell, supplying more than $95 \%$ of the ATP for metabolism [43]. Thus, mitochondria are a logical point to define the pathophysiological processes and therapeutic targets in various metabolic diseases. To explore the relationship between the heart and kidney, we established 
the renal IR rat model by removing the right kidney and clamping the left renal artery for 30 min followed by reperfusion for $24 \mathrm{~h}$. We then pre-treated the rats with TIIA combined with CsA to determine mitochondrial function. As shown in Figure 4, renal IRI induced AMI, and the electron microscopic photographs $(40,000 \times)$ of myocardial tissue revealed that renal IRI induced abnormal mitochondrial morphology and increased the percentage of damaged mitochondria, such as mitochondrial swelling and membrane rupture, particularly in the obese model rats. TIIA+ CsA alleviated the mitochondrial injury (Figure 2B). Oxygen consumption rate and RCR decreased in the IR and IR (obese) groups, particularly in the IR (obese) group, compared with the sham group $(p<0.05)$. Pretreatment with TIIA, CsA, and TIIA+CsA alleviated the decrease in the oxygen consumption rate and RCR $(p<0.05)$ (Figure 4A). Renal IR significantly increased the mitochondrial ROS level and opening of the MPAP $(\%)(p<0.05)$. TIIA, CsA, and TIIA+CsA significantly decreased the mitochondrial ROS level and opening of the MPAP $(\%)(p<$ 0.05 ) (Figure 4A). Renal IR decreased the MMP (ratio of red/green) significantly $(p<0.05)$, whereas TIIA, CsA, and TIIA+CsA decreased the mitochondrial MMP (ratio of red/green) significantly $(p<0.05)$ (Figure $4 A)$. These results are similar to those of Luan Get al [44].

The mtDNA copy number in each mitochondrion is stable, so the total copy number of mtDNA can be used to estimate the quantity of mitochondria [45]. Although the mtDNA repair mechanism is unknown, mtDNA is near the respiratory chain, so it is more vulnerable when exposed to oxidative reactions, compared to nuclear DNA. In our study, we observed the damaging effect of renal IR on mtDNA and the protective effect of TIIA+CsA, using the ratio of long and short fragments to assess the degree of damage. Our results show that the ratio of long/short fragments decreased in the IR and IR (obese) group, particularly in the IR (obese) group, compared with the sham, TIIA, CsA, and TIIA+CsA groups ( $p<$ 0.05), which increased after pretreatment with TIIA, CsA, and TIIA+CsA $(p<0.05)$ (Figure 4A). As mtDNA was damaged by renal IR, synthesis of the complex proteins in the mitochondrial electron transport chain would be inhibited, resulting in obstruction of electron transport.

Respiration is one of the most vital and basic features of living organisms. In mammals, respiration is accomplished by respiratory chain complexes located on the mitochondrial inner membrane to transfer electrons and establish the proton gradient for complex $V$ to synthesize ATP [46]. As shown in Figure 4B,

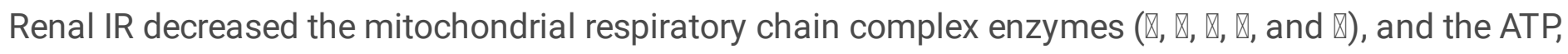
particularly in the IR (obese) group. Pretreatment with TIIA, CsA, and TIIA+CsA significantly increased these enzymes.

As an important adaptation to exposure to chronic energy deprivation, mitochondrial biogenesis is modulated by many complex elements, such as Tfam and Nrf1. Nrf1 fosters transcription of nucleiencoded mitochondrial protein expression, including factors involved in the respiratory complexes and oxidative phosphorylation. Tfam increases gene transcription and DNA replication in mitochondria by directly binding to the mitochondrial genome. PGC-1a is an important transcriptional co-activator that modulates key factors, including Nrf1 and Tfam, which increase mitochondrial biogenesis [47]. Mitochondrial biogenesis becomes chaotic when expression of these gene changes. As shown in Figure 5, renal IR reduced the expression of PGC-1a, Nrf1, and Tfam. TIIA+CsA markedly increased the levels of 
PGC-1a, Nrf1, and Tfam. After the TIIA+CsA treatment, increased mitochondrial oxygen consumption rate, a sufficient energy supply (ATP), decreased ROS, and improved mitochondria biogenesis factors acted synergistically to improve the intracellular energy supply shortage. Harmful stimuli, including oxidative stress, aging, and energy limitations damage mitochondria, which become fused to lysosomes and degraded. Autophagic abnormalities in mitochondria increase the number of damaged mitochondria [44].

Mitochondria normally undergo a dynamic process of fusion and fission. This dynamic process is important to maintain constant changes in size, shape, and network of mitochondria. Mitochondria are under the control of regulatory proteins, including Drp1 and Mfns [48]. Our results (Figure 5) show that the expression of Mfns (Mfn1 and Mfn2) and Drp1 changed after renal IR, showing a chaotic balance of fission-fusion in mitochondria. Mfns are believed to have an important role in mitochondrial fusion, and Drp1 may have an important role in mitochondrial fission. We observed increases in Mfn1 and Mfn2, and a decrease in Drp1 in myocardial tissues after renal IR.

The PI3K/Akt/Bad signaling pathway plays an important role inhibiting apoptosis mediated by mitochondria [15]. This pathway can regulate the expression of downstream apoptosis proteins (Bax and $\mathrm{Bcl}-2$ ), and it can regulate the cell migration, growth, angiogenesi, invasion [49]. Activiting the PI3K-AKT signalling pathway can inhibit apoptosis through dephosphorylating phosphatidylinositol triphosphate to phosphatidylinositol diphosphate [50]. Because of the close relationship between them, we researched the effect of PI3K in this study. PI3K is a phosphatidylinositol kinase with activities as a serine/threoninespecific protein kinase and a phosphatidylinositol kinase [51]. After activation, phosphatidylinositol family members are phosphorylated on the cell membrane and the downstream signal molecule Akt is recruited and activated. Activated Akt phosphorylates the Ser136/Ser112 residues of the Bad protein [52]. Phosphorylated Bad separates from the apoptosis-promoting complex and forms the 14-3-3 protein complex, leading to inactivation of its apoptosis-promoting function, which inhibits apoptosis [53]. Our results show that TIIA+CsA effectively regulated the expression of apoptotic PI3K/Akt/Bad pathwayrelated proteins, and TIIA+CsA enhanced PI3K and p-Akt expression but downregulated expression of Cytc, caspase-9/3, and PARP (Figure 6). These results show that TIIA+CsA adjusted mitochondrial function and inhibited apoptosis of myocardial cells induced by renal IRI by activating the PI3K/Akt/Bad pathway.

In our study, we used isolated mitochondria to show that renal IR promotes the production of ROS, which damages mtDNA, mitochondrial respiratory function, biogenesis, and dynamic function. Swollen mitochondria were induced by opening of the MPTP after renal IR. Opening of the MPTP induced back flow of protons from the mitochondrial membrane space into the matrix, thereby reducing ATP synthesis and MMP, leading to metabolic abnormalities. A reduction of MMP and ATP synthesis and an increase of Cyt-c were induced by opening of the MPTP, which led to back flow of protons from the mitochondrial membrane space to the matrix to induce metabolic abnormalities and eventually leading to myocardial cell apoptosis. The TIIA+CsA pre-treatment inhibited apoptosis by modulating mitochondrial function through the $\mathrm{PI3K} / \mathrm{Akt} / \mathrm{Bad}$ pathway in obese rats. But in our study, considering of the combination therapy, we did not use the PI3K/Akt/Bad pathway inhibitor, so the mechanism of drug intervention can 
not be fully revealed,in the future, we will use the LY294002 (PI3K inhibitor) in the in vitro cell experiment in order to fully reveal the mechanism of its intervention in the future.

\section{Conclusions}

Renal IR can induce the mitochondrial dysfunction and apoptosis (obesity increases the severity) in myocardial cell, TIIA combined with CsA can attenuated myocardial cell apoptosis by modulating mitochondrial function through the $\mathrm{PI} 3 \mathrm{~K} / \mathrm{Akt} /$ Bad pathway in obese rats.

\section{Abbreviations}

IR, ischemia-reperfusion; AKI, acute kidney injury; AMI, acute myocardial injury; CsA, cyclosporine A; TIIA, Tanshinone IIA; HE, hematoxylin-eosin; TUNEL, terminal deoxynucleotidyl transferase-mediated dUTP nick end-labeling; MMP, mitochondrial membrane potential; ATP, adenosine triphosphate; ROS, reactive oxygen species; RCR, respiration controlling rate; $\mathrm{MPTP}$, mitochondrial permeability transition pore; CVD, cardiovascular diseases; CyP-D, cyclophilin D; DMSO, dimethylsulfoxide; HFD, high-fat diet; SD, SpragueDawley; PBS, phosphate buffer saline; CK-MB, creatine kinase isoenzymes-muscle/brain; cTNI, cardiac troponin I; IL-1 $\beta$, interleukin-1 $\beta$; TNF- $\alpha$, tumor nerosis factors- $\alpha$; TM, time-motion; LVIDs, left ventricular end-systolic internal diameter; LVIDd, left ventricular end-diastolic internal diameter; EF, ejection fraction; FS, fractional shortening; BCA, bicinchoninic acid; OD, optical density; DAPI, 4',6-diamidino-2-phenylindole; EDTA, ethylene diamine tetraacetic acid; BCA, bicinchoninic acid; DCFH-DA, 2',7'-

Dichlorodihydrofluorescein diacetate; RT-qPCR, Real-time quantitative Polymerase Chain Reaction; ANOVA, one-way analysis of variance; SDS-PAGE, sodium dodecyl sulfate-polyacrylamide gel electrophoresis; IRI, ischemia-reperfusion injury; ELISA, euzymelinked immunosorbent assay; PI3K, Phosphoinositide-3 kinase; Cyt-C, cytochrome C; PARP, poly ADP-ribose polymerase; Drp1, dynamin-related protein 1; Mfns, Mitofusins; PGC-1a, PPARy coactivator-1-a; PGC-1a, PPARy coactivator-1-a; Nrf1, Nucleo respiratory factor 1 ; Tfam, Transcription factor $A$ of mitochondrial

\section{Declarations}

\section{Acknowledgements}

The authors would like to thank all of the rats, the team of investigators, research partners, and operations staff involved in this study. We want to thank professor Lian-qun Jia for scientific.

\section{Conflicts of interest: none declared.}

\section{Funding}

The study was supported by the National Natural Science Foundation of China (81774022); Open Fund Project of Key Laboratory of Ministry of Education for Traditional Chinese Medicine Visera-State Theory and Application (xyzx1906). Liaoning Natural Science Foundation of China (20180551242). Inheritance 
and Innovation Project of Traditional Chinese Medicine (Qihuang Project), and "Xingliao Yingcai Project" of Liaoning Province

\section{Availability of data and materials}

The datasets generated and analyzed during the current study are available from the corresponding author on reasonable request

\section{Authors' contributions}

He Tai and Xiao-lin Jiang wrote the manuscript and researched data; Yue Li, Hong-he Xiao, Si-cheng Yao, and Nan Song selected the rats and extracted blood; Mei-jun Lv and Jin Wu dealed with the figures; Wei Chen, Jia-xiang Pan, and Xin-yue Cui detected related index; Guan-lin Yang, Zhi-ming Lan and Lian-qun Jia contributed to the discussion and reviewed the manuscript. Rats model is builded by Si-cheng Yao and Mei-jun Lv. Editorial support (in the form of writing assistance, including development of the initial draft based on author input, assembling tables and figures, collating authors comments, grammatical editing and referencing) was provided by He Tai and Lian-qun Jia. The translator of English was provided by He Tai. Finally, I would like to thank my wife Xiao-lin Jiang for her support and understanding of my scientific research work.

\section{Ethics approval and consent to participate}

Animal experimental design and husbandry procedures were approved by the Ethical Committee of Animal Handling (2019019) at Liaoning University of Traditional Chinese Medicine and complied with the guidelines of the Use and Care of laboratory animals published by US National Institutes of Health. We did our best to decrease the number of rats and suffering during the study.

\section{Consent for publication}

All listed authors consent to the submission and all data are used with the consent of the person generating the data.

\section{Competing interests}

The authors declare that they have no competing interests.

\section{Author details}

${ }^{1}$ Key Laboratory of Ministry of Education for Traditional Chinese Medicine Viscera-State Theory and Applications, Liaoning University of Traditional Chinese Medicine, Huanggu District Chongshan Road No. 79, Shenyang, Liaoning, 110847, China.

${ }^{2}$ School of Pharmacy, Liaoning University of Traditional Chinese Medicine, 77 Life One Road, DD Port, Dalian, Liaoning, 116600, China. 
${ }^{3}$ Department of Cardiovascular Medicine, The Affiliated Hospital of Liaoning Traditional Chinese Medicine, Huanggu District Beiling Road No. 33, Shenyang, Liaoning, 110032, China.

${ }^{4}$ Department of Endocrinology and Metabolic, Shenyang the Fourth Hospital of People, Huanggu District Huanghe Road No. 20, Shenyang, Liaoning, 110031, China.

${ }^{5}$ Department of Medical laboratory, The fourth of Affiliated Hospital of Guangzhou University of

Traditional Chinese Medicine (Shenzhen Traditional Chinese Medicine Hospital), Guangzhou University of Traditional Chinese Medicine, Futian District Fuhua Road No. 1, Shenzhen, Guangdong, 518021, China.

\section{References}

[1] Koyner JL, Cerdá J, Goldstein SL, Jaber BL, Liu KD, Shea JA, Faubel S. The daily burden of acute kidney injury: a survey of US nephrologists on world kidney day. Am J Kidney Dis. 2014; 64 (3): 394-401. https://doi.org/10.1053/j.ajkd.2014.03.018.

PMID: 24815216

[2] Oztay F, Kara-Kisla B, Orhan N, Yanardag R, Bolkent S. The protective effects of prostaglandin E1 on lung injury following renal ischemia-reperfusion in rats. Toxicol Ind Health. 2016; 32(9): 1684-1692. https://doi.org/10.1177/0748233715576615.

PMID: 25883098

[3] Hafner S, Hillenbrand A, Knippschild U, Radermacher P. The obesity paradox and acute kidney injury: beneficial effects of hyper-inflammation? Crit Care. 2013; 10; 17 (6): 1023.

https://doi.org/10.1186/cc13152.

PMID: 24326122

[4] Kelz RR, Reinke CE, Zubizarreta JR, Wang M, Saynisch P, Even-Shoshan O, et al. Acute kidney injury, renal function, and the elderly obese surgical patient: a matched case-control study. Ann Surg. 2013; 258 (2): 359-363.

https://doi.org/10.1097/SLA.0b013e31829654f3.

PMID: 23676533

[5] Harmankaya O, Akalin N, Akay H, Okuturlar Y, Erturk K, Kaptanogullari H, Kocoglu H. Comparison of risk factors for cardiovascular disease in hemodialysis and peritoneal dialysis patients. Clinics (Sao Paulo). 2015;70 (9): 601-605. 
https://doi.org/10.6061/clinics/2015(09)01.

PMID: 26375560

[6] Panico K, Abrahão MV, Trentin-Sonoda M, Muzi-Filho H, Vieyra A, Carneiro-Ramos MS. Cardiac Inflammation after Ischemia-Reperfusion of the Kidney: Role of the Sympathetic Nervous System and the Renin-Angiotensin System. Cell Physiol Biochem. 2019; 53 (4): 587-605.

https://doi.org/10.33594/000000159.

PMID: 31535830

[7] Cirino-Silva R, Kmit FV, Trentin-Sonoda M, Nakama KK, Panico K, Alvim JM, Dreyer TR, Martinho-Silva $\mathrm{H}$, Carneiro-Ramos MS. Renal ischemia/reperfusion-induced Cardiac Hypertrophy in Mice: Cardiac Morphological and Morphometric Characterization. JRSM Cardiovasc Dis. 2017; 1; 6: 2048004016689440.

https://doi.org/10.1177/2048004016689440.

PMID: 28228941

[8] Kwong JQ, Molkentin JD. Physiological and pathological roles of the mitochondrial permeability transition pore in the heart. Oxid Med Cell Longev.Cell Metab. 2015; 21 (2): 206-214.

https://doi.org/10.1016/j.cmet.2014.12.001.

PMID: 25651175

[9] Yin X, Yin Y, Cao FL, Chen YF, Peng Y, Hou WG, Sun SK, Luo ZJ. Tanshinone IIA attenuates the inflammatory response and apoptosis after traumatic injury of the spinal cord in adult rats. Plos One. 2012; 7 (6): e38381.

https://doi.org/10.1371/journal.pone.0038381.

PMID: 22675554

[10] Xu W, Yang J, Wu L M. Cardioprotective effects of tanshinone IIA on myocardial ischemia injury in rats. Die Pharmazie. 2009; 64 (5): 332-336.

PMID: 19530445

[11] Zhang SZ, Ye ZG, Xia Q, Zhang W, Bruce I. Inhibition of Mitochondrial Permeability Transition Pore: A Possible Mechanism for Cardioprotection Conferred by Pretreatment with Tanshinone IIA. Conf Proc IEEE Eng Med Biol Soc. 2005; 3: 2276-2279.

https://doi.org/10.1109/IEMBS.2005.1616918. 
[12] Baines CP, Kaiser RA, Purcell NH, Blair NS, Osinska H, Hambleton MA, Brunskill EW, Sayen MR, Gottlieb RA, Dorn GW, Robbins J, Molkentin JD. Loss of cyclophilin D reveals a critical role for mitochondrial permeability transition in cell death. Nature. 2005 Mar 31; 434 (7033): 658-662.

https://doi.org/10.1038/nature03434.

PMID: 20700952

[13] Lemoine S, Pillot B, Augeul L, Rabeyrin M, Varennes A, Normand G, Baetz D, Ovize M, JuillardL. Dose and timing of injections for effective cyclosporine A pretreatment before renal ischemia reperfusion in mice. PloS One. 2017; 10; 12 (8): e0182358.

https://doi.org/10.1371/journal.pone.0182358.

PMID: 28796779

[14] Rodgers SJ, Ferguson DT, Mitchell CA, Ooms LM. Regulation of PI3K effector signalling in cancer by the phosphoinositide phosphatases. Biosci Rep. 2017; 10;37 (1): BSR20160432.

https://doi.org/10.1042/BSR20160432.

PMID: 28082369

[15] KW Zeng, XM Wang, H Ko, HC Kwon, JW Cha, HO Yang. Hyperoside Protects Primary Rat Cortical Neurons From Neurotoxicity Induced by Amyloid $\beta$-protein via the PI3K/Akt/Bad/Bcl(XL)-regulated Mitochondrial Apoptotic Pathway. Eur J Pharmacol. 2011; 672 (1-3): 45-55.

https://doi.org/10.1016/j.ejphar.2011.09.177.

PMID: 21978835

[16] Neto JS, Nakao A, Kimizuka K, Romanosky AJ, Stolz DB, Uchiyama T, Nalesnik MA , Otterbein LE, Murase N. Protection of transplantinduced renal ischemia-reperfusion injury with carbon monoxide. Am Physiol J Renal Physiol. 2004; 287 (5): F979-989.

https://doi.org/10.1152/ajprenal.00158.2004.

PMID: 15292046

[17] Alzoubi KH, Abdul-Razzak KK, Khabour OF, Al-Tuweiq GM, Alzubi MA, Alkadhi KA. Adverse effect of combination of chronic psychosocial stress and high fat diet on hippocampus-dependent memory in rats. Behav Brain Res. 2009; 204 (1): 117-123.

https://doi.org/10.1016/j.bbr.2009.05.025. 
PMID: 19482049

[18] Tawfik MK. Renoprotective activity of telmisartan versus pioglitazone on ischemia/reperfusion induced renal damage in diabetic rats. Eur Rev Med Pharmacol Sci. 2012; 16 (5): 600-609.

PMID: 22774400

[19] Ali SI, Alhusseini NF, Atteia HH, Idris RA, Hasan RA3. Renoprotective effect of a combination of garlic and telmisartan against ischemia/reperfusion-induced kidney injury in obese rats. Free Radic Res. 2016; 50 (9): 966-986.

https://doi.org/10.1080/10715762.2016.1211644.

PMID: 27405440

[20] Han A, Lu Y, Zheng Q, Zhang J, Zhao Y, Zhao M, Cui X. Qiliqiangxin Attenuates Cardiac Remodeling via Inhibition of TGF- $\beta 1 /$ Smad3 and NF-KB Signaling Pathways in a Rat Model of Myocardial Infarction. Cell Physiol Biochem. 2018; 45 (5): 1797-1806.

https://doi.org/10.1159/000487871.

PMID: 29510381

[21] Zhou J, Ma X, Shi M, Chen C, Sun Y, Li J, Xiong Y, Chen J, Li F. Serum metabolomics analysis reveals that obvious cardioprotective effects of low dose Sini decoction against isoproterenol-induced myocardial injury in rats. Phytomedicine. 2017; 15; 31: 18-31.

https:// 10.1016/j.phymed.2017.01.009.

PMID: 28606513

[22] Lv H, Yu F, Sha C, Huang Y, Lu Y, Zhang L, Zhai R, Wang T, Fu F. Effects of rotigotine and rotigotine extended-release microsphere therapy on myocardial ischemic injury in mice. Pharm Sci. 2019;134:1-6.

https:// 10.1016/j.ejps.2019.04.009.

PMID: 30959104

[23] Al-Amran F, Shahkolahi M. Oxytocin ameliorates the immediate myocardial injury in rat heart transplant through downregulation of neutrophil-dependent myocardial apoptosis. Transplant Proc. 2013; 45 (6): 2506-2512.

https:// 10.1016/j.transproceed.2013.03.022

PMID: 23953571 
[24] Luan G, Li G, Ma X, Jin Y, Hu N, Li J, Wang ZH, Wang HL. Dexamethasone-Induced Mitochondrial Dysfunction and Insulin Resistance-Study in 3T3-L1 Adipocytes and Mitochondria Isolated from Mouse Liver. Molecules. 2019; 23; 24 (10): pii: E1982.

https://doi.org/10.3390/molecules24101982.

PMID: 31126054

[25] Li X, Jia P, Huang Z, Liu S, Miao J, Guo Y, Wu N, Wu N, Jia DL. Lycopene Protects Against Myocardial Ischemia-Reperfusion Injury by Inhibiting Mitochondrial Permeability Transition Pore Opening. Drug Des Devel Ther. 2019; 11;13:2331-2342.

https://doi.org/10.2147/DDDT.S194753.

PMID: 31371925

[26] Gilmer LK, Ansari MA, Roberts KN, Scheff SW. Age-Related Mitochondrial Changes after Traumatic Brain Injury. J Neurotrauma. 2010; 27 (5): 939-950.

https://doi.org/10.1089/neu.2009.1181.

PMID: 20175672

[27] Federica C, Rosalba S, Pasquale L, Angela Z, Arianna M, Raffaella C, Giovanna L, Antonia L, Fernando G, Susanna I. Fructose-Rich Diet Affects Mitochondrial DNA Damage and Repair in Rats. Nutrients. 2017; 24; 9 (4): 323.

https: //doi.org/0.3390/nu9040323.

PMID: 28338610

[28] Wagner EM. Monitoring gene expression: quantitative real-time RT-PCR. Methods Mol Biol. 2013; 1027: $19-45$.

\section{https://doi.org/10.1007/978-1-60327-369-5_2.}

PMID: 23886954

[29] Livak KJ, Schmittgen TD. Analysis of relative gene expression data using real-time quantitative PCR and the 2(-Delta DeltaC(T)) Method. Methods. 2001; 25 (4): 402-408.

https://doi.org/10.1006/meth.2001.1262.

PMID: 11846609 
[30] Chieko M, May KHS, Miniwan T, Qi Y, Tokujiro U, Shinya Abe, Masanobu K, Satoshi I, Yoshinobu E, Makoto T. Effects of atrial natriuretic peptide on inter-organ crosstalk among the kidney, lung, and heart in a rat model of renal ischemia-reperfusion injury. Intensive Care Med Exp. 2014; 2 (1): 28.

https://doi.org/10.1186/s40635-014-0028-8.

PMID: 26266925

[31] Sousou IA, Naglaa FA, Hebatallah HA, Reham AEI-SI, Rehab AH. Renoprotective Effect of a Combination of Garlic and Telmisartan Against Ischemin/Reperfusion-Induced Kidney Injury in Obse Rats. Free Radic Res. 2016; 50 (9): 966-986.

https://doi.org/10.1080/10715762.2016.1211644.

PMID: 27405440

[32] Agarwal KC. Therapeutic actions of garlic constituents. Med Res Rev. 1996;16 (1):111-124.

https://doi.org/10.1002/(SICI)1098-1128(199601)16:1<111::AID-MED4>3.0.C0;2-5.

PMID: 8788216

[33] Hassoun HT, Lie ML, Grigoryev DN, Liu M, Tuder RM, Rabb H. Kidney ischemia-reperfusion injury induces caspase-dependent pulmonary apoptosis. Am J Physiol Renal Physiol. 2009; 297 (1): F125-137. https://doi.org/10.1152/ajprenal.90666.2008.

PMID: 19403643

[34] Jang HR, Ko GJ, Wasowska BA, Rabb H. The interaction between ischemia-reperfusion and immune responses in the kidney. J Mol Med (Berl). 2009; 87 (9): 859-864.

https://doi.org/10.1007/s00109-009-0491-y.

PMID: 19562316

[35] Schrier RW. Role of diminished renal function in cardiovascular mortality: marker or pathogenetic factor? J Am Coll Cardiol. 2006; 3; 47 (1): 1-8.

https://doi.org/10.1016/j.jacc.2005.07.067.

PMID: 16386657

[36] Vasan RS, Sullivan LM, Roubenoff R, Dinarello CA, Harris T, Benjamin EJ, Sawyer DB, Levy D, Willson PWF, D'Agostini RB, Framingham Heart Study. Inflammatory markers and risk of heart failure in elderly 
subjects without prior myocardial infarction: the Framingham Heart Study. Circulation. 2003; 25; 107 (11):1486-1491.

https://doi.org/10.1161/01.cir.0000057810.48709.f6.

PMID: 12654604

[37] Cirino-Silva R, Kmit FV, Trentin-Sonoda M, Nakama KK, Panico K, Alvim JM, Dreyer TR, Martinho-Silva $\mathrm{H}$, Carneiro-Ramos MS. Renal ischemia/reperfusion-induced cardiac hypertrophy in mice: Cardiac morphological and morphometric characterization. JRSM Cardiovasc Dis. 2017; 6: 2048004016689440.

https://doi.org/10.1177/2048004016689440.

PMID:28228941

[38] Li JJ, Chen Q, He XH, Azeem A, NingJL, Bin Y, Lu KZ, Gu JT. Dexmedetomidine Attenuates Lung Apoptosis Induced by Renal Ischemia-Reperfusion Injury Through a2AR/PI3K/Akt Pathway. J Transl Med. 2018; 23;16 (1): 78.

https://doi.org/10.1186/s12967-018-1455-1.

PMID: 29566706

[39] Xu YM, Ding GH, Huang J, Xiong Y. Tanshinone IIA Pretreatment Attenuates Ischemia/ReperfusionInduced Renal Injury. Exp Ther Med. 2016;12 (4): 2741-2746.

https://doi.org/10.3892/etm.2016.3674.

PMID: 27698779

[40] Zhang Z, He H, Qiao Y, Huang J, Wu Z, Xu P, Yin D, He M. Tanshinone IIA Pretreatment Protects H9c2 Cells Against Anoxia/Reoxygenation Injury: Involvement of the Translocation of Bcl-2 to Mitochondria Mediated by 14-3-3 ๆ. Oxid Med Cell Longev. 2018; 28; 2018: 3583921.

https://doi.org/10.1155/2018/3583921.

PMID: 30050654

[41] Lemoine S, Pillot B, Augeul L, Rabeyrin M, Varennes A, Normand G, Baetz D, Ovize M, Juillard L. Dose and timing of injections for effective cyclosporine A pretreatment before renal ischemia reperfusion in mice. PLoS One. 2017; 10; 12 (8): e0182358.

https://doi.org/10.1371/journal.pone.0182358.

PMID: 28796779 
[42] Lemoine S, Pillot B, Rognant N, Augeul L, Rayberin M, Varennes A, Laville M, Ovize M, Juillard L. Postconditioning with cyclosporine a reduces early renal dysfunction by inhibiting mitochondrial permeability transition. Transplantation. 2015; 99 (4):717-723.

https://doi.org/10.1097/TP.0000000000000530.

PMID: 25793558

[43] M Kaaman, L M Sparks, V van Harmelen, S R Smith, E Sjölin, I Dahlman, P Arner. Strong association between mitochondrial DNA copy number and lipogenesis in human white adipose tissue. Diabetologia. 2007, 50 (12), 2526-2533.

https://doi.org/10.1007/s00125-007-0818-6.

PMID: 17879081

[44] Luan G, Li G, Ma X, Jin Y, Hu N, Li J, Wang ZH, Wang HL. Dexamethasone-Induced Mitochondrial Dysfunction and Insulin Resistance-Study in 3T3-L1 Adipocytes and Mitochondria Isolated from Mouse Liver. Molecules. 2019; 23; 24 (10): pii: E1982.

https://doi.org/10.3390/molecules24101982.

PMID: 31126054

[45] Federica C, Rosalba S, Pasquale L, Angela Z, Arianna M, Raffaella C, Giovanna L, Antonia L, Fernando G, Susanna I. Fructose-Rich Diet Affects Mitochondrial DNA Damage and Repair in Rats. Nutrients. 2017; 24; 9 (4): 323.

https: //doi.org/10.3390/nu9040323.

PMID: 28338610

[46] Wu M, Gu J, Guo R, Huang Y, Yang M. Structure of Mammalian Respiratory Supercomplex I(1)III(2)IV(1). Cell. 2016; 167 (6): 1598-1609.

https: //doi.org/10.1016/j.cell.2016.11.012.

PMID: 27912063

[47] Kelly DP, Scarpulla RC. Transcriptional regulatory circuits controlling mitochondrial biogenesis and function. Genes Dev. 2004, 15; 18 (4): 357-368.

https://doi.org/10.1101/gad.1177604.

PMID: 15004004 
[48] de Brito OM, Scorrano L. Mitofusin 2 tethers endoplasmic reticulum to mitochondria. Nature. 2008, 4; 456 (7222): 605-610.

https://doi.org/10.1038/nature07534.

PMID: 19052620

[49] Long ZW, Wu JH, Cai-Hong, Wang YN, Zhou Y. Mir-374b promotes proliferation and inhibits apoptosis of human gist cells by inhibiting pten through activation of the pi3k/akt pathway. Mol Cells. 2018;41 (6): 532-544.

https://doi.org/10.14348/molcells.2018.2211.

PMID: 29902839

[50] Jiang Y, Chang H, Chen G. Effects of microRNA-20a on the proliferation, migration and apoptosis of multiple myeloma via the PTEN/PI3K/AKT signaling pathway. Oncol Lett. 2018;15 (6): 10001-10007. https://doi.org/10.3892/ol.2018.8555.

PMID: 29963125

[51] Wang YL, Yuan YT, Gao YT, Li X, Tian F, Liu F, Du Rc, Li PF, Wang F, Xu SM, Wu XQ, Wang CF. MicroRNA-31 regulating apoptosis by mediating the phosphatidylinositol-3kinase/protein kinase $B$ signaling pathway in treatment of spinal cord injury. Brain Dev. 2019, 41 (8): 649-661.

https://doi.org/10.1016/j.braindev.2019.04.010.

PMID: 31036380

[52] Fang X, Yu S, Eder A, Mao M, Bast RC Jr, Boyd D, Mills GB. Regulationof BAD phosphorylation at serine 112 by the Ras-mitogen-activated protein kinase pathway. Oncogene. 1999, 18 (48): 6635-6640. https://doi.org/10.1038/sj.onc.1203076.

PMID: 10597268

[53] Sakamaki J, Daitoku H, Katsuya U, Ayano H, Kazuyuki Y, Akiyoshi F. Arginine Methylation of BCL-2 Antagonist of Cell Death (BAD) Counteracts Its Phosphorylation and Inactivation by Akt. Proc Natl Acad Sci USA. 2011, 12;108 (15): 6085-6090.

https://doi.org/10.1073/pnas.1015328108.

PMID: 21444773 


\section{Tables}

Table.1 Sequence of primers for RT-PCR and long PCR 


\begin{tabular}{|c|c|c|c|}
\hline Target Gene & Primer Sequence & Size (bp) & $\operatorname{Tm}\left({ }^{\circ} \mathrm{C}\right)$ \\
\hline \multirow[t]{2}{*}{ Mfn1 } & Forward: 5'-GGGAAGACCAAATCGACAGA-3' & \multirow[t]{2}{*}{152} & 57 \\
\hline & Reverse: 5'-CAAAACAGACAGGCGACAAA-3' & & 57 \\
\hline \multirow[t]{2}{*}{ Mfn2 } & Forward: 5'-GAGAGGCGATTTGAGGAGTG-3' & \multirow[t]{2}{*}{165} & 58 \\
\hline & Reverse: 5'-CTCTTCCCGCATTTCAAGAC-3' & & 56 \\
\hline \multirow[t]{2}{*}{ Drp1 } & Forward: 5'-GCCCGTGGATGATAAAAGTG-3' & \multirow[t]{2}{*}{215} & 56 \\
\hline & Reverse: 5'-TGGCGGTCAAGATGTCAATA-3' & & 56 \\
\hline \multirow[t]{2}{*}{ PGC-1a } & Forward: 5'-GGACGAATACCGCAGAGAGT-3' & \multirow[t]{2}{*}{201} & 59 \\
\hline & Reverse: 5'-CCATCATCCCGCAGATTTAC-3' & & 56 \\
\hline \multirow[t]{2}{*}{ Nrf1 } & Forward: 5'-AAACCGAACACATGGCTACC-3' & \multirow[t]{2}{*}{168} & 58 \\
\hline & Reverse: 5'-CTGCCGTGGAGTTGAGTATG-3' & & 58 \\
\hline \multirow[t]{2}{*}{ Tfam } & Forward: 5'-TCACCTCAAGGGAAATTGAAG-3' & \multirow[t]{2}{*}{241} & 55 \\
\hline & Reverse: 5'-CCCAATCCCAATGACAACTC-3' & & 56 \\
\hline \multirow[t]{2}{*}{ Long Fragment } & Forward:5'-AAAATCCCCGCAAACAATGACCACCC-3' & \multirow[t]{2}{*}{13400} & 72 \\
\hline & Reverse: 5'-GGCAATTAAGAGTGGGATGGAGCCAA-3' & & 72 \\
\hline \multirow{2}{*}{$\begin{array}{l}\text { Shrot } \\
\text { Fragment }\end{array}$} & Forward: 5'-CСТCCCATTCATTATCGCCGCCCTGC-3' & \multirow[t]{2}{*}{235} & 60 \\
\hline & Reverse: 5'-GTCTGGGTCTCCTAGTAGGTCTGGGAA-3' & & 60 \\
\hline \multirow[t]{2}{*}{ Bax } & Forward: 5'-GCGATGAACTGGACAACAAC-3' & \multirow[t]{2}{*}{200} & 57 \\
\hline & Reverse: 5'-GATCAGCTCGGGCACTTTAG-3' & & 58 \\
\hline \multirow[t]{2}{*}{$\mathrm{Bcl}-2$} & Forward: 5'-CGAGTGGGATACTGGAGATGA-3' & \multirow[t]{2}{*}{236} & 58 \\
\hline & Reverse: 5'- GACGGTAGCGACGAGAGAAG-3' & & 59 \\
\hline \multirow[t]{2}{*}{ Caspase-3 } & Forward: 5'-CCCATCACAATCTCACGGTAT-3' & \multirow[t]{2}{*}{195} & 57 \\
\hline & Reverse: 5'-GGACGGAAACAGAACGAACA-3' & & 58 \\
\hline \multirow[t]{2}{*}{ Caspase-9 } & Forward: 5'-GCCTCTGCTTTGTCATGGAG-3' & \multirow[t]{2}{*}{181} & 56 \\
\hline & Reverse: 5'-AGCATGAGGTTCTCCAGCTT-3' & & 56 \\
\hline \multirow[t]{2}{*}{ PI3K } & Forward: 5'-TCACCTCCCTGATTGGCTAC-3' & \multirow[t]{2}{*}{220} & 58 \\
\hline & Reverse: 5'-CCACGATGGATGACAATGAA-3' & & 55 \\
\hline \multirow[t]{2}{*}{ Akt } & Forward: 5'-CGAGTCCCCACTCAACAACT-3' & \multirow[t]{2}{*}{231} & 59 \\
\hline & Reverse: 5'-GGTGAACCTGACCGGAAGTC-3' & & 60 \\
\hline
\end{tabular}




\begin{tabular}{|c|c|c|c|}
\hline \multirow[t]{2}{*}{ Bad } & Forward: 5'-GAGCTGACGTACAGCGTTGA-3' & \multirow[t]{2}{*}{153} & 60.39 \\
\hline & Forward: 5'-CCTGAGGGCTGTCCAGTAAC-3' & & 59.75 \\
\hline \multirow[t]{2}{*}{ PARP } & Forward: 5'-AAGCCTGGCACTAAGTCGAA-3' & \multirow[t]{2}{*}{164} & 59.31 \\
\hline & Forward: 5'-ATAGAGTAGGCGGCCTGGAT-3' & & 59.88 \\
\hline \multirow[t]{2}{*}{ Сус-с } & Forward: 5'-GGACAGCCCCGATTTAAGTA-3' & \multirow[t]{2}{*}{121} & 57 \\
\hline & Forward: 5'-TCAATAGGTTTGAGGCGACAC-3' & & 58 \\
\hline \multirow[t]{2}{*}{ GAPDH } & Forward: 5'- AGGTCGGTGTGAACGGATTTG -3' & \multirow[t]{2}{*}{20} & 58 \\
\hline & Reverse: 5'- GGGGTCGTTGATGGCAACA-3' & & 58 \\
\hline
\end{tabular}

\section{Table.2 Antibodies used in the study}




\begin{tabular}{|lllll|}
\hline Antibodies & Manufacturer & Catalogue No. & Observed MW & Dilution \\
\hline Anti-PI3K & Proteintech & $67071-1-1 \mathrm{~g}$ & $110 \mathrm{KDa}$ & $1: 10,000$ \\
\hline Anti-p-Akt & Proteintech & $66444-1-1 \mathrm{~g}$ & $62 \mathrm{KDa}$ & $1: 10,000$ \\
\hline Anti-Akt & Proteintech & $10176-2-\mathrm{AP}$ & $56 \mathrm{KDa}$ & $1: 5,000$ \\
\hline Anti-p-Bad & Cell Signaling Technology & $5284 \mathrm{~S}$ & $23 \mathrm{KDa}$ & $1: 1,000$ \\
\hline Anti-Bad & Proteintech & $10435-1-\mathrm{AP}$ & $18 \mathrm{KDa}$ & $1: 2,500$ \\
\hline Anti-Bcl-2 & Proteintech & $26593-1-\mathrm{AP}$ & $26 \mathrm{KDa}$ & $1: 2,500$ \\
\hline Anti-Bax & Proteintech & $50599-2-1 \mathrm{~g}$ & $26 \mathrm{KDa}$ & $1: 10,000$ \\
\hline Anti-Caspase-3 & Proteintech & $19677-1-\mathrm{AP}$ & $32 \mathrm{KDa}$ & $1: 2,000$ \\
\hline Anti-cleaved-Caspase-3 & Abcam & ab49822 & $17 \mathrm{KDa}$ & $1: 500$ \\
\hline Anti-Caspase-9 & Proteintech & $10380-1-\mathrm{AP}$ & $47 \mathrm{KDa}$ & $1: 1,000$ \\
\hline Anti-cleaved-Caspase-9 & Affinity Biosciences & AF5240 & $10 \mathrm{KDa}$ & $1: 1,000$ \\
\hline Anti-PARP1 & Proteintech & $13371-1-A P$ & $89 \mathrm{KDa}$ & $1: 2,000$ \\
\hline Anti-Cyt-c & Proteintech & $12245-1-A P$ & $13 \mathrm{KDa}$ & $1: 2,000$ \\
\hline Anti-Mfn1 & Proteintech & $13798-1-A P$ & $86 \mathrm{KDa}$ & $1: 1,000$ \\
\hline Anti-Mfn2 & Proteintech & $12186-1-A P$ & $86 \mathrm{KDa}$ & $1: 5,000$ \\
\hline Anti-Drp1 & Proteintech & $10656-1-A P$ & $27 \mathrm{KDa}$ & $1: 4,000$ \\
\hline Anti-PGC1a & Proteintech & $66369-1-1 \mathrm{~g}$ & $100 \mathrm{KDa}$ & $1: 5,000$ \\
\hline Anti-Nrf1 & Proteintech & $12482-1-A P$ & $67 \mathrm{KDa}$ & $1: 2,500$ \\
\hline Anti-Tfam & Proteintech & $25 \mathrm{KDa}$ & $1: 5,000$ \\
\hline Anti-GAPDH & Proteintech & $36 \mathrm{KDa}$ & $1: 10,000$ \\
\hline
\end{tabular}

Table. 3 The cause of death in the six groups rats 


\begin{tabular}{|lllllll|}
\hline Cause of death & Sham & IR (non) & $\begin{array}{l}\text { IR } \\
\text { (obese) }\end{array}$ & TIIA & CsA & TIIA+CsA \\
\hline Infection after injection & 00 & 0 & 0 & 1 & 0 & 0 \\
\hline Massive haemorrhage & 1 & 1 & 2 & 2 & 3 & 3 \\
\hline $\begin{array}{l}\text { Infection after surgery } \\
\text { Intestinal obstruction }\end{array}$ & 2 & 2 & 3 & 3 & 2 & 3 \\
$\begin{array}{l}\text { Number of completed } \\
\text { cases (n (\%)) }\end{array}$ & 13 & 4 & 5 & 4 & 4 & 5 \\
\hline
\end{tabular}

\section{Figures}



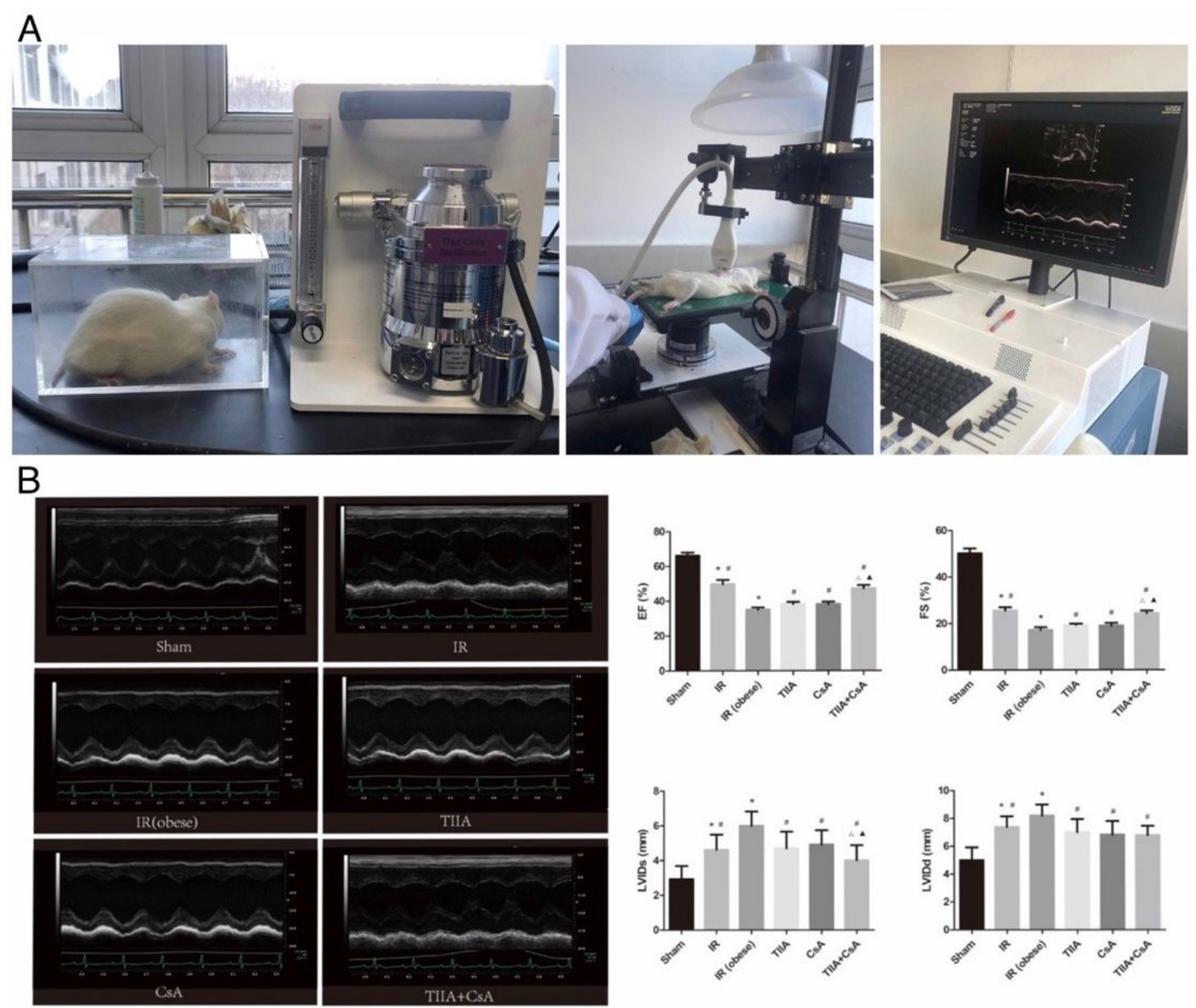

C
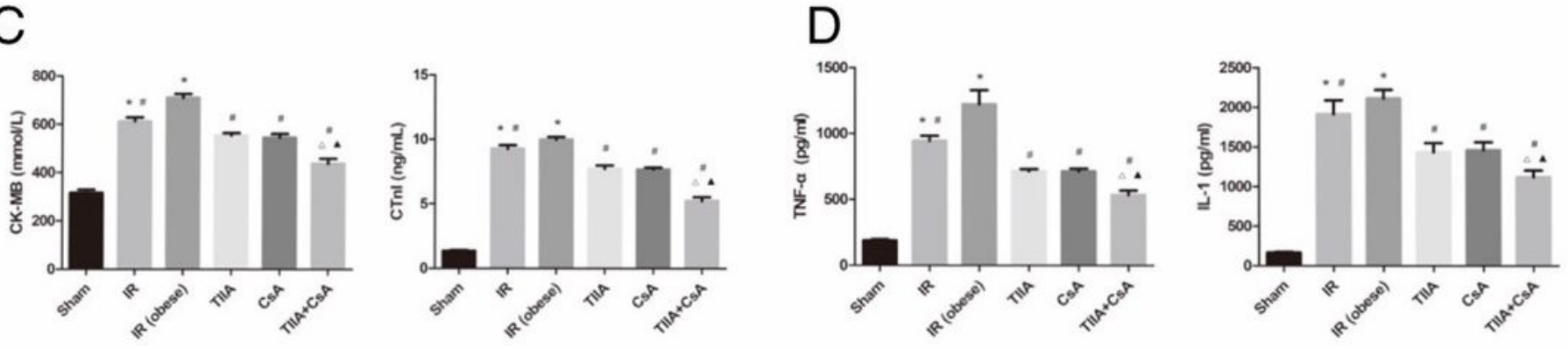

\section{Figure 1}

Tanshinone IIA (TIIA)+ cyclosporine A (CsA) improved the cardiac function, myocardial enzyme, and inflammatory factor after renal ischemia-reperfusion (IR) Rats were pre-treated with TIIA alone or in combination with CsA followed by removing the right kidney and clamping of the left renal artery for 30 min and reperfusion for $24 \mathrm{~h}$. Sham rats were used as control. Cardiac function (B), myocardial enzyme (C), and inflammatory factor (D) were evaluated under different groups. Data are shown as mean \pm SD. *p 
$<0.05$ versus sham group, $\# p<0.05$ versus IR (obese) group, $\triangle p<0.05$ versus TIIA group, $\Delta p<0.05$ versus CsA group.

A

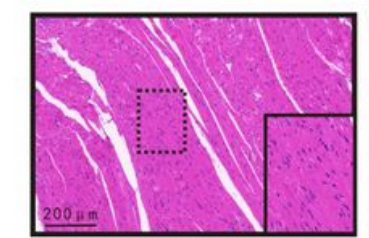

Sham

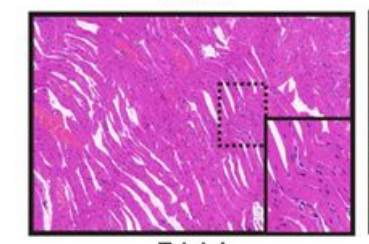

TIIA

B

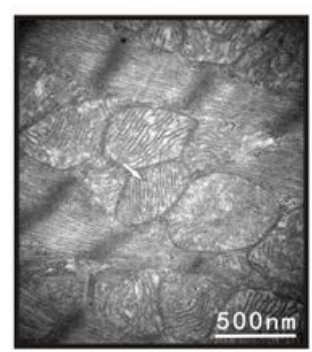

Sham

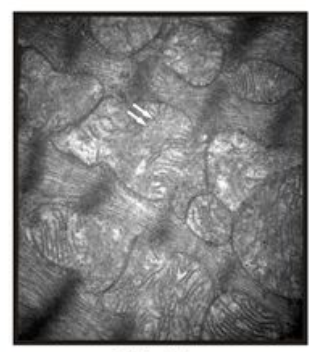

TI I A

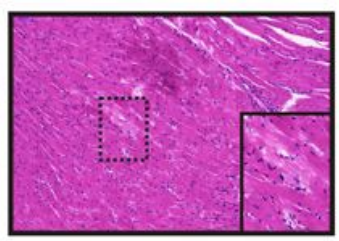

IR

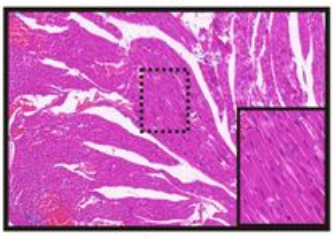

CsA

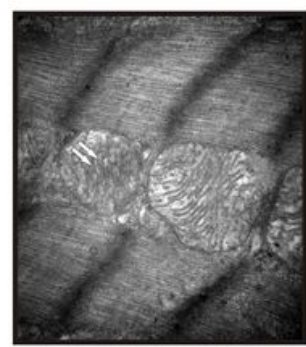

IR

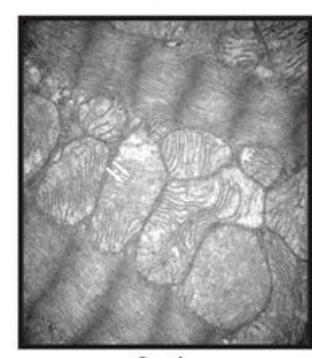

CsA

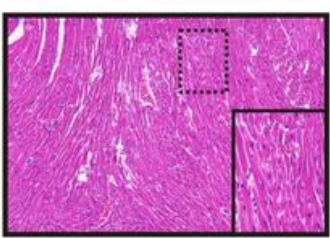

IR (obese)

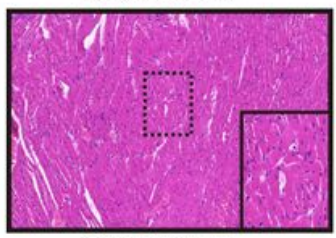

$\mathrm{TI} \mid \mathrm{A}+\mathrm{CsA}$

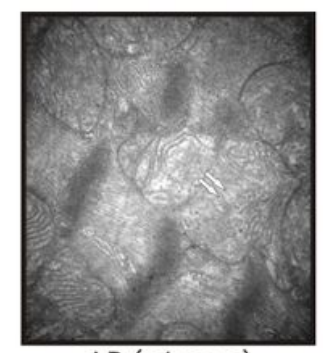

IR (obese)

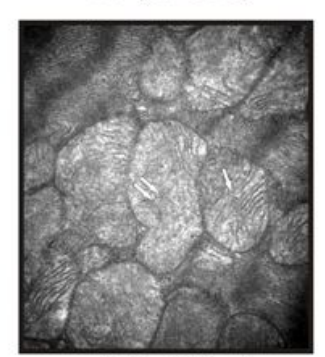

$\mathrm{T} I \mathrm{I}+\mathrm{CsA}$

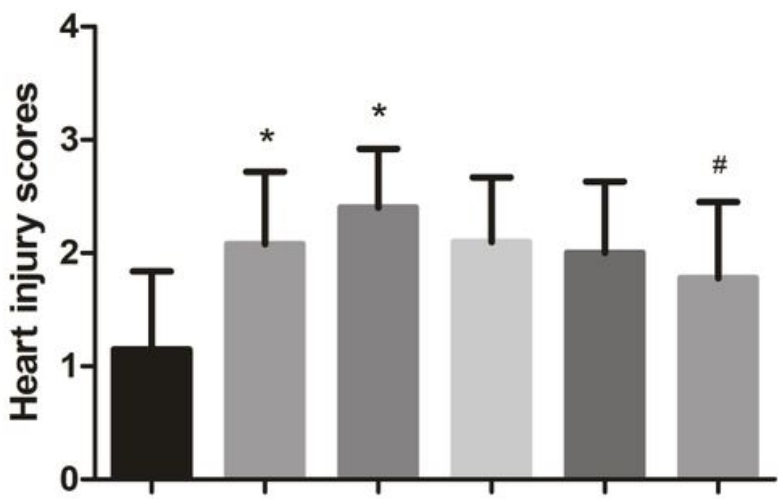

son

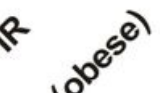

s
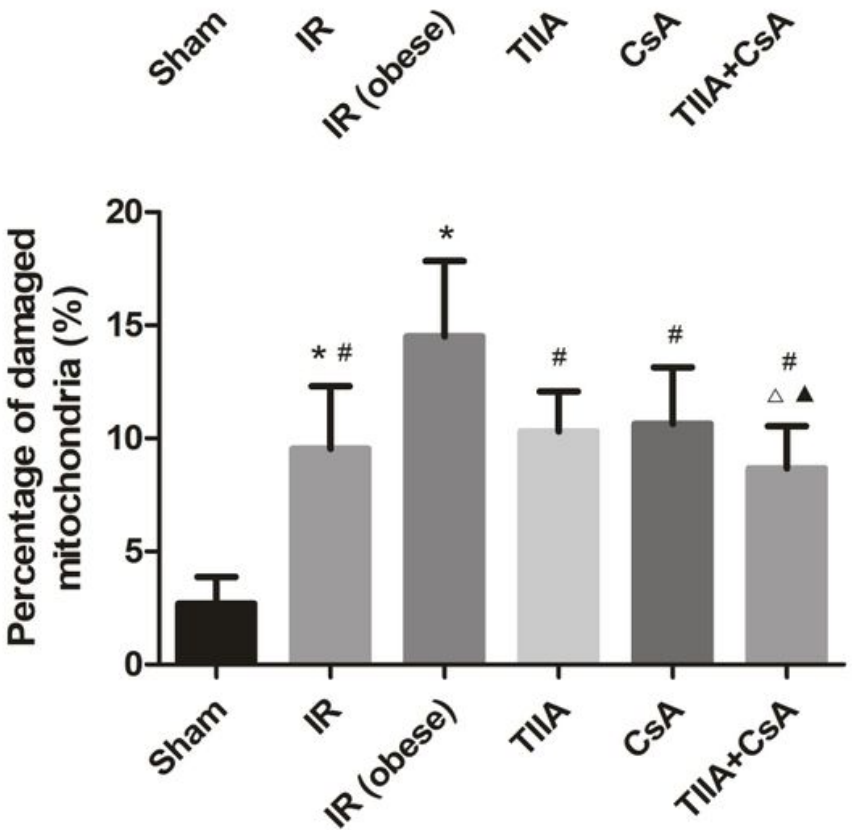

Figure 2

Tanshinone IIA (TIIA)+ cyclosporine A (CsA) preserved myocardial architecture in renal ischemiareperfusion (IR)-induced myocardial injury Rats were pre-treated with TIIA alone or in combination with CsA followed by removing the right kidney and clamping of the left renal artery for 30 min and reperfusion for $24 \mathrm{~h}$. Sham rats were used as control. Representative photomicrographs of myocardial histology (A), the scale bars represents a length of $200 \mu \mathrm{m}$ on histology, myocardial injury scores (A) were evaluated under different conditions. The scale bars represents a length of $200 \mu \mathrm{m}$ on histology. Electron microscope pictures (40 000x) of rats myocardial tissue after renal ischemia-reperfusion (IR). Abnormal mitochondrial (paired white arrow) morphology show that mitochondrial membrane rupture or swellings, normal mitochondrial (single white arrow) morphology type show that mitochondrial membrane smooth and inner carinulae distinct (B), and percentage of damaged mitochondria (B). Data are shown as mean \pm SD. ${ }^{*} p<0.05$ versus sham group, $\# p<0.05$ versus IR (obese) group, $\triangle p<0.05$ versus TIIA group, $\Delta p<$ 
0.05 versus CsA group. Data are shown as mean \pm SD. ${ }^{*} p<0.05$ versus sham group, \#p $<0.05$ versus IR (obese) group, $\triangle p<0.05$ versus TIIA group, $\Delta p<0.05$ versus CsA group.

A
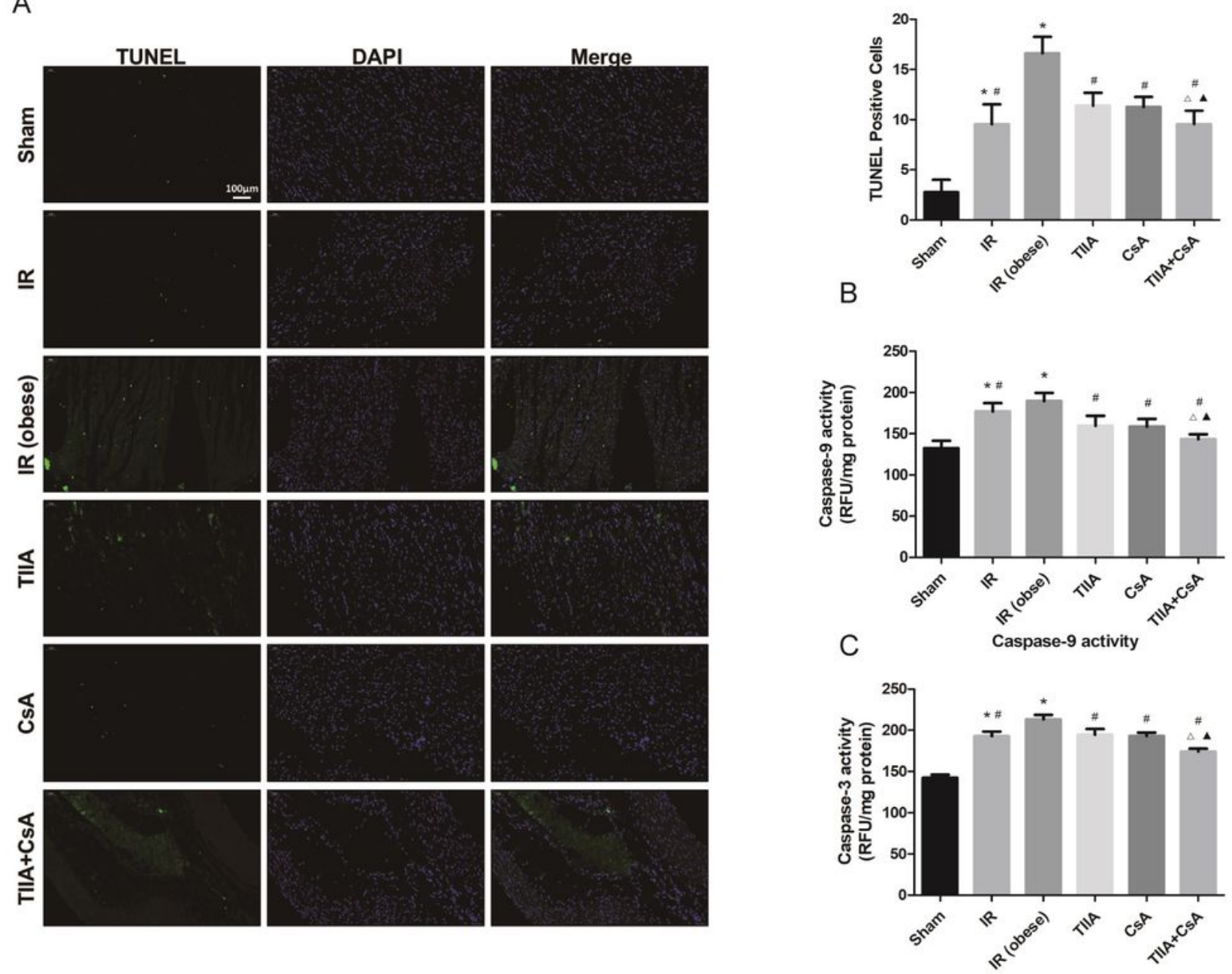

D

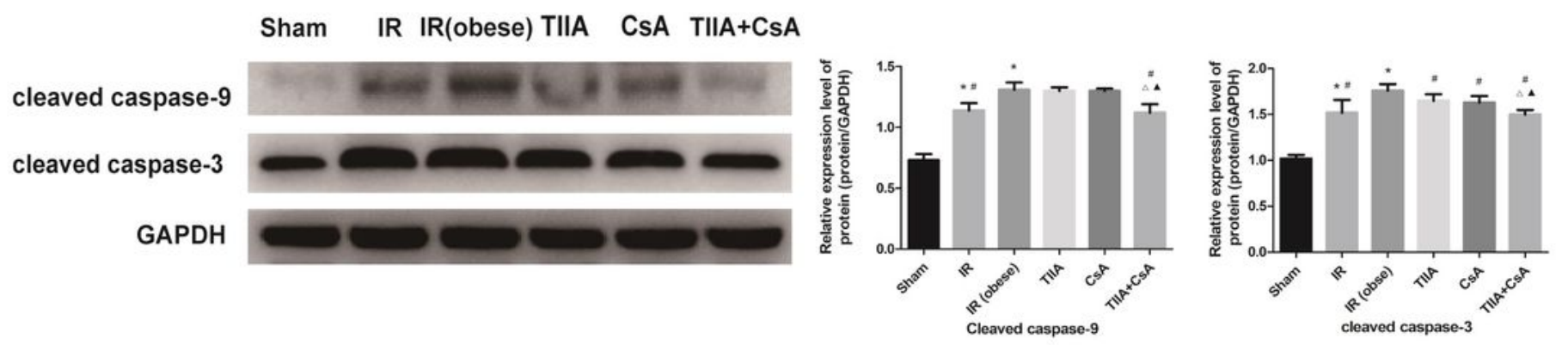

Figure 3

Tanshinone IIA (TIIA)+ cyclosporine A (CsA) inhibited myocardial cells apoptosis after renal ischemiareperfusion (IR) Rats were pre-treated with TIIA alone or in combination with CsA followed by removing the right kidney and clamping of the left renal artery for 30 min and reperfusion for $24 \mathrm{~h}$. Sham rats were 
used as control. Representative apoptosis of myocardial cells (A), TUNEL positive cells (A), the scale bars represents a length of $100 \mu \mathrm{m}$ on histology, the activity of myocardial caspase-9/3 (B, C), and the protein expression of cleaved caspase-9/3 (D) were evaluated under different groups. Data are shown as mean \pm SD. ${ }^{\star} p<0.05$ versus sham group, $\# p<0.05$ versus IR (obese) group, $\triangle p<0.05$ versus TIIA group, $\Delta p<$ 0.05 versus CsA group.

A
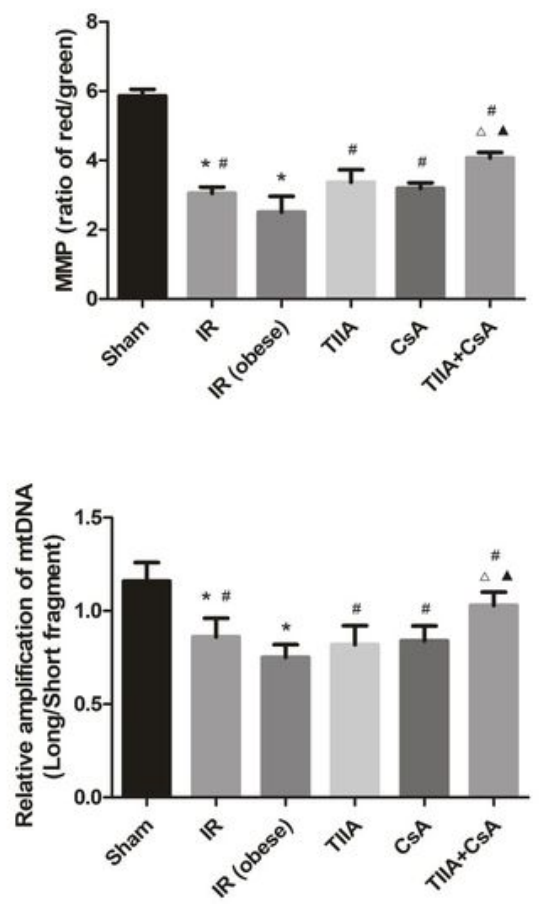

B
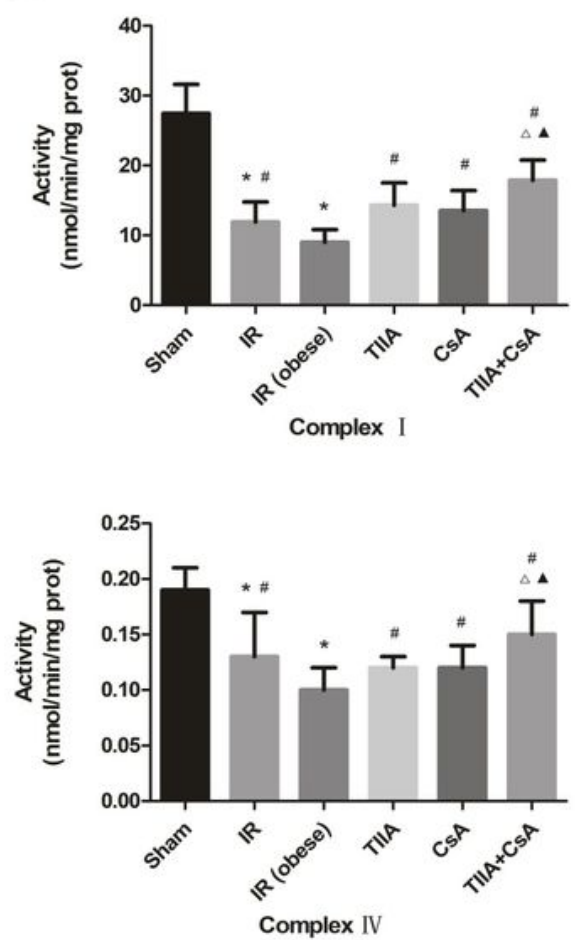
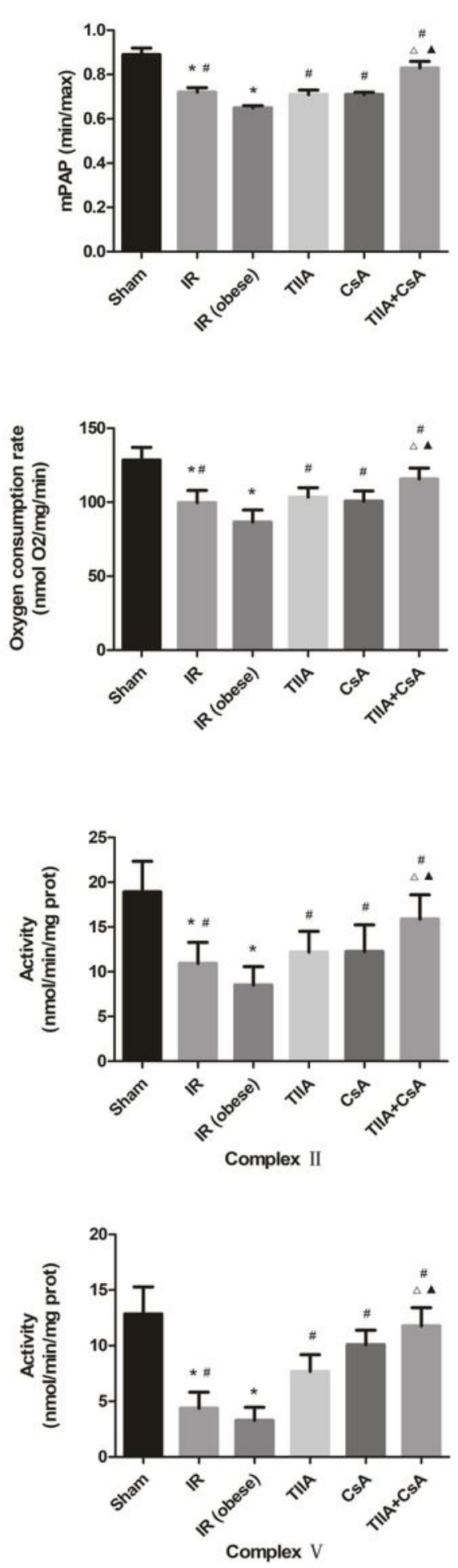
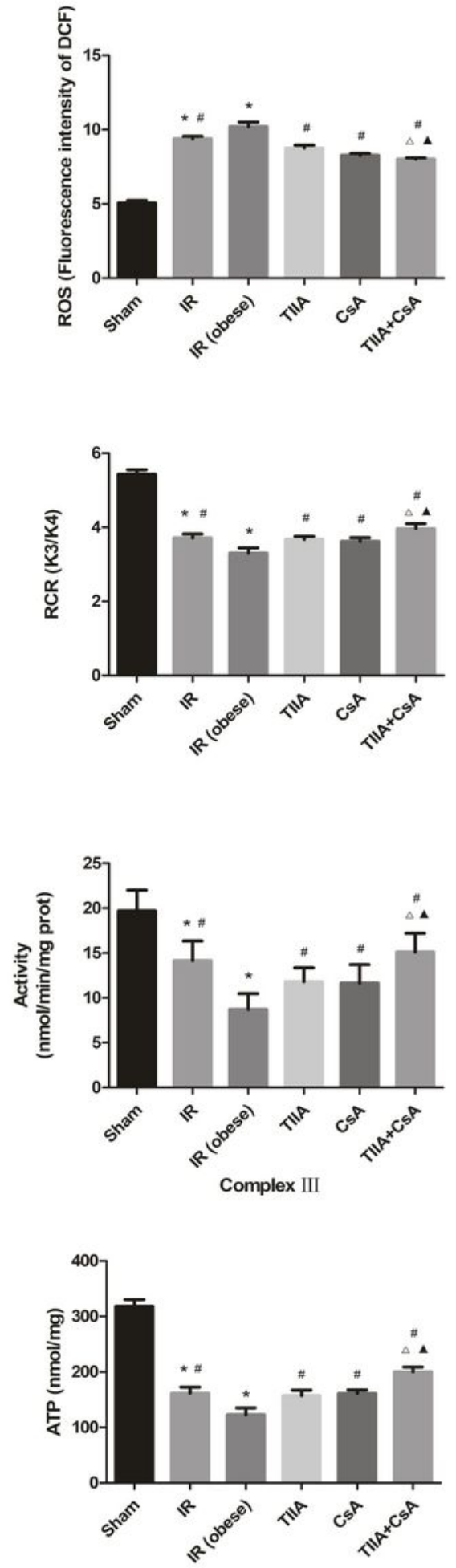

Figure 4 
Tanshinone IIA (TIIA)+ cyclosporine A (CsA) preserved myocardial mitochondrial function in renal ischemia-reperfusion (IR)-induced myocardial injury The MMP (ratio of red/green), the opening of mPAP (\%), the mitochondrial ROS, the mtDNA damage (ratio of long/short fragments), the mitochondrial RCR, mitochondrial oxygen consumption rate $(A)$, the mitochondrial respiratory chain complex enzymes $(\mathbb{\nabla}, \mathbb{Q}, \mathbb{\nabla}$, $\bigotimes$, and $\otimes)$, and ATP $(B)$ were recorded above. Rats were pre-treated with TIIA alone or in combination with CsA followed by removing the right kidney and clamping of the left renal artery for $30 \mathrm{~min}$ and reperfusion for $24 \mathrm{~h}$. Sham rats were used as control. ${ }^{*} p<0.05$ versus sham group, $\# p<0.05$ versus IR (obese) group, $\triangle p<0.05$ versus TIIA group, $\Delta p<0.05$ versus CsA group. 
A
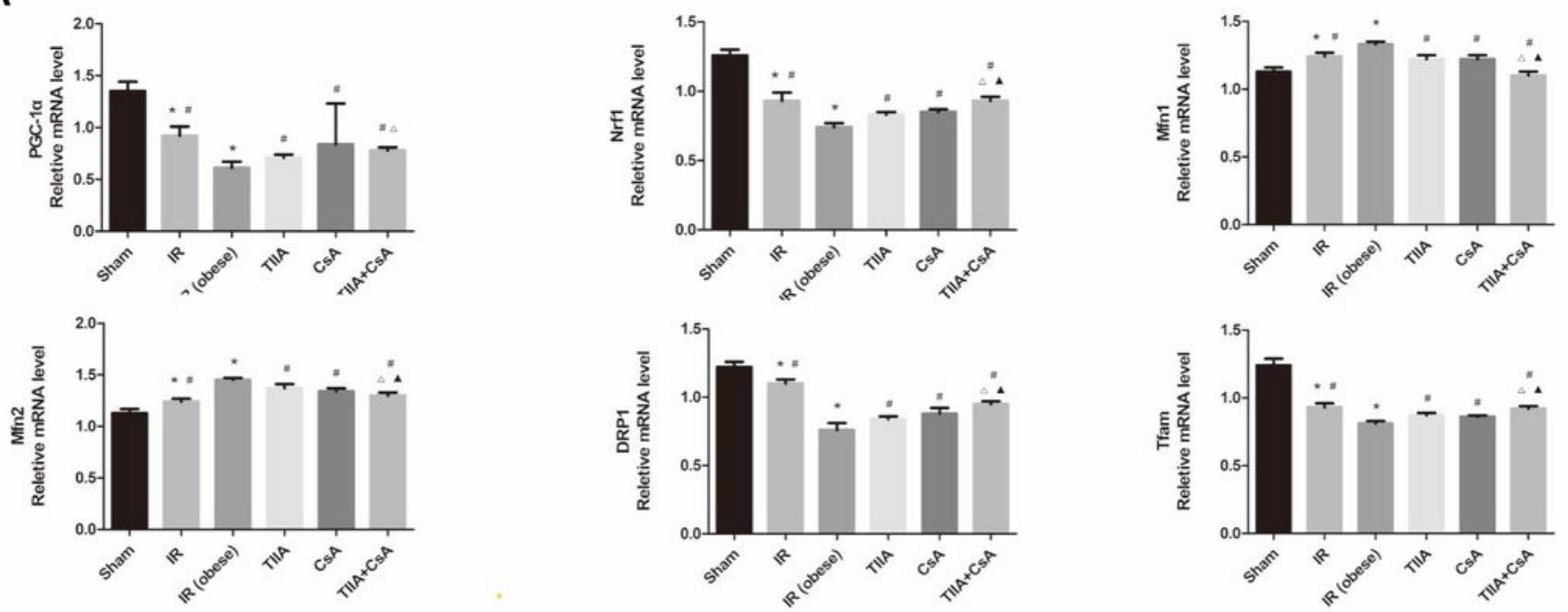

B

\section{Sham IR IR(obese) TIIA CsA TIIA+CsA}
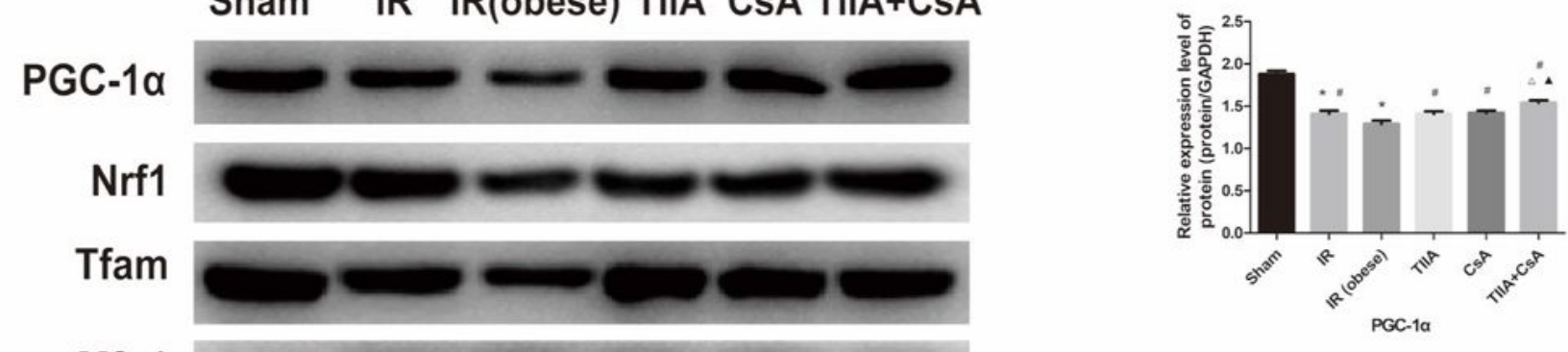

Mfn1

Mfn2

Drp1

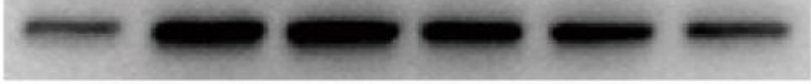

GAPDH
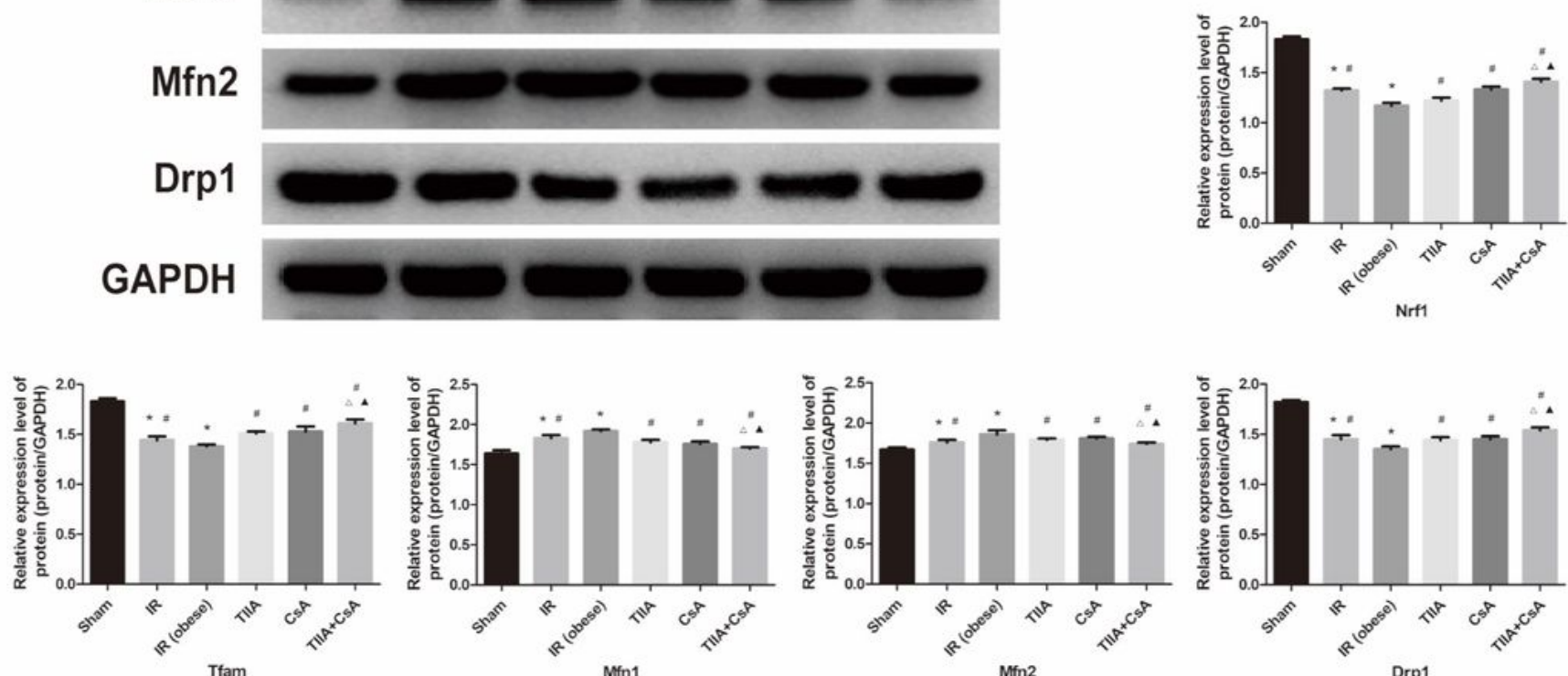

Min1

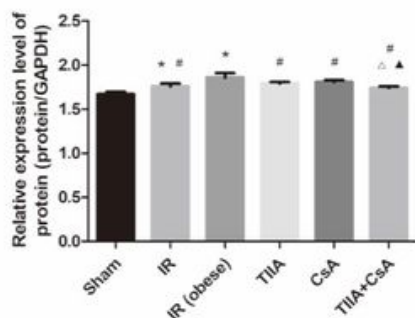

Mfn2

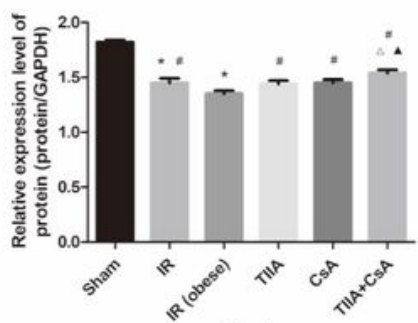

Drp1

\section{Figure 5}

Tanshinone IIA (TIIA)+ cyclosporine A (CsA) preserved mitochondrial biogenesis and dynamics in renal renal ischemia-reperfusion (IR)-induced myocardial injury The expression of PGC-1a, Nrf1, and Tfam in mRNA (A) and protein (B) level. The expression of Mfn1, Mfn2, and Drp1 in mRNA and protein level level. ${ }^{*} p<0.05$ versus sham group, $\# p<0.05$ versus IR (obese) group, $\triangle p<0.05$ versus TIIA group, $\Delta p<0.05$ versus CsA group. 
A
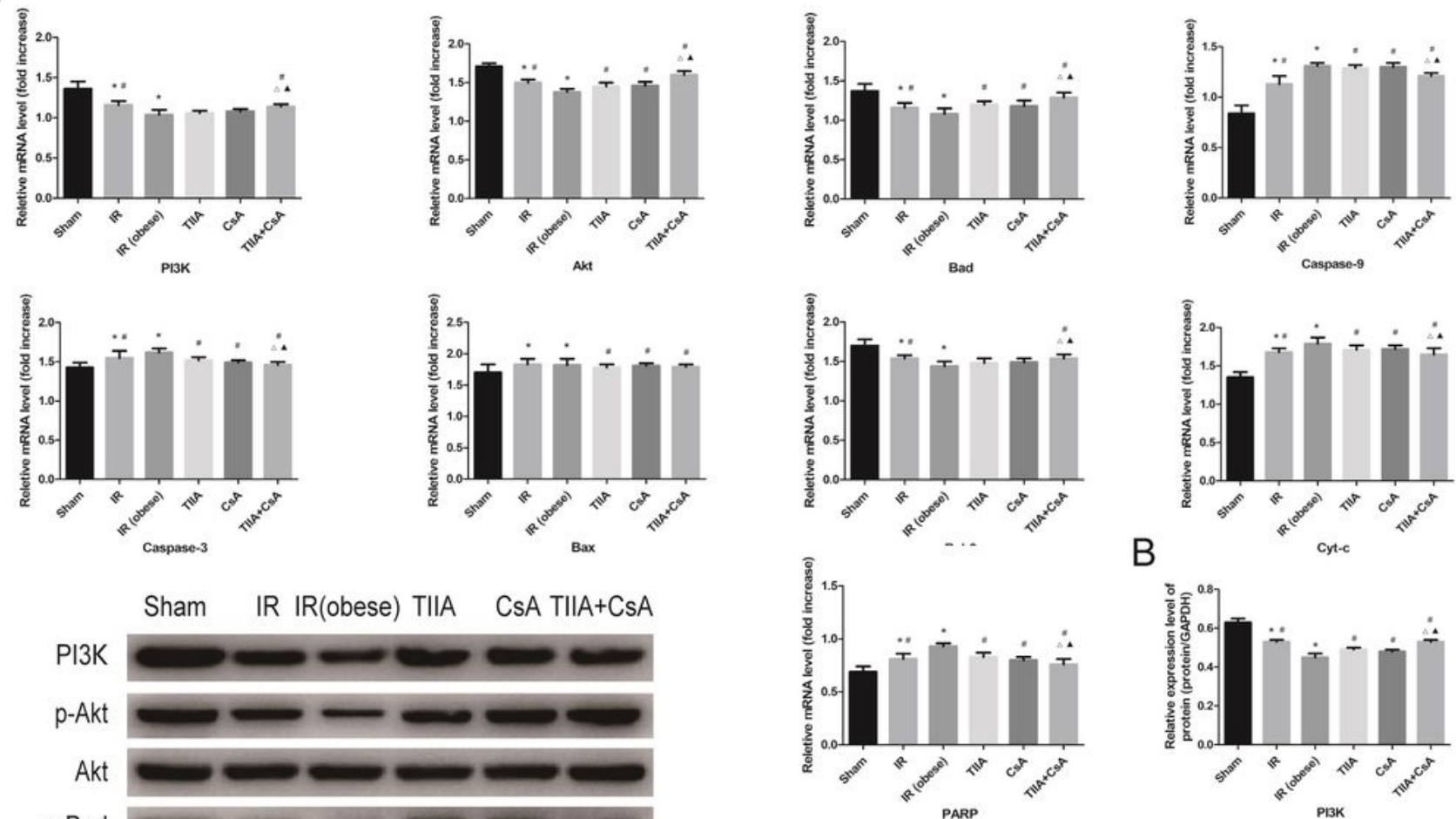

$\mathrm{B}$

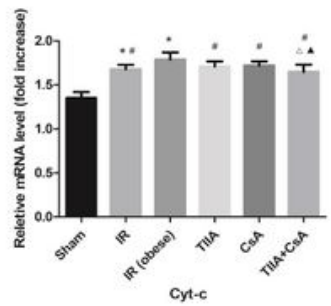

Sham IR IR(obese) TIIA CsA TIIA+CsA

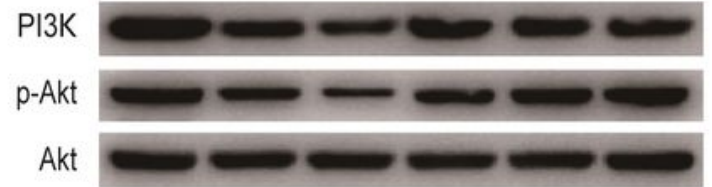

p-Bad

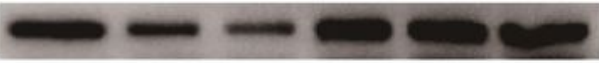

Bad

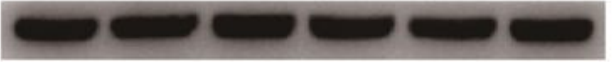

caspase- 9

caspase-3

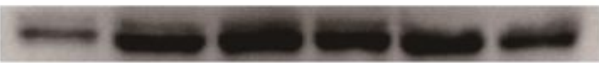

Bax

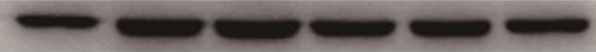

$\mathrm{Bcl}-2$

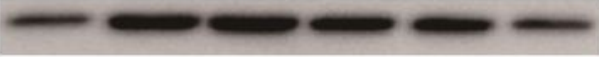

Cyt-c

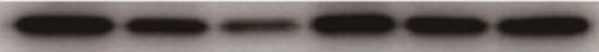

PARP

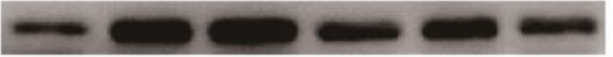

GAPDH
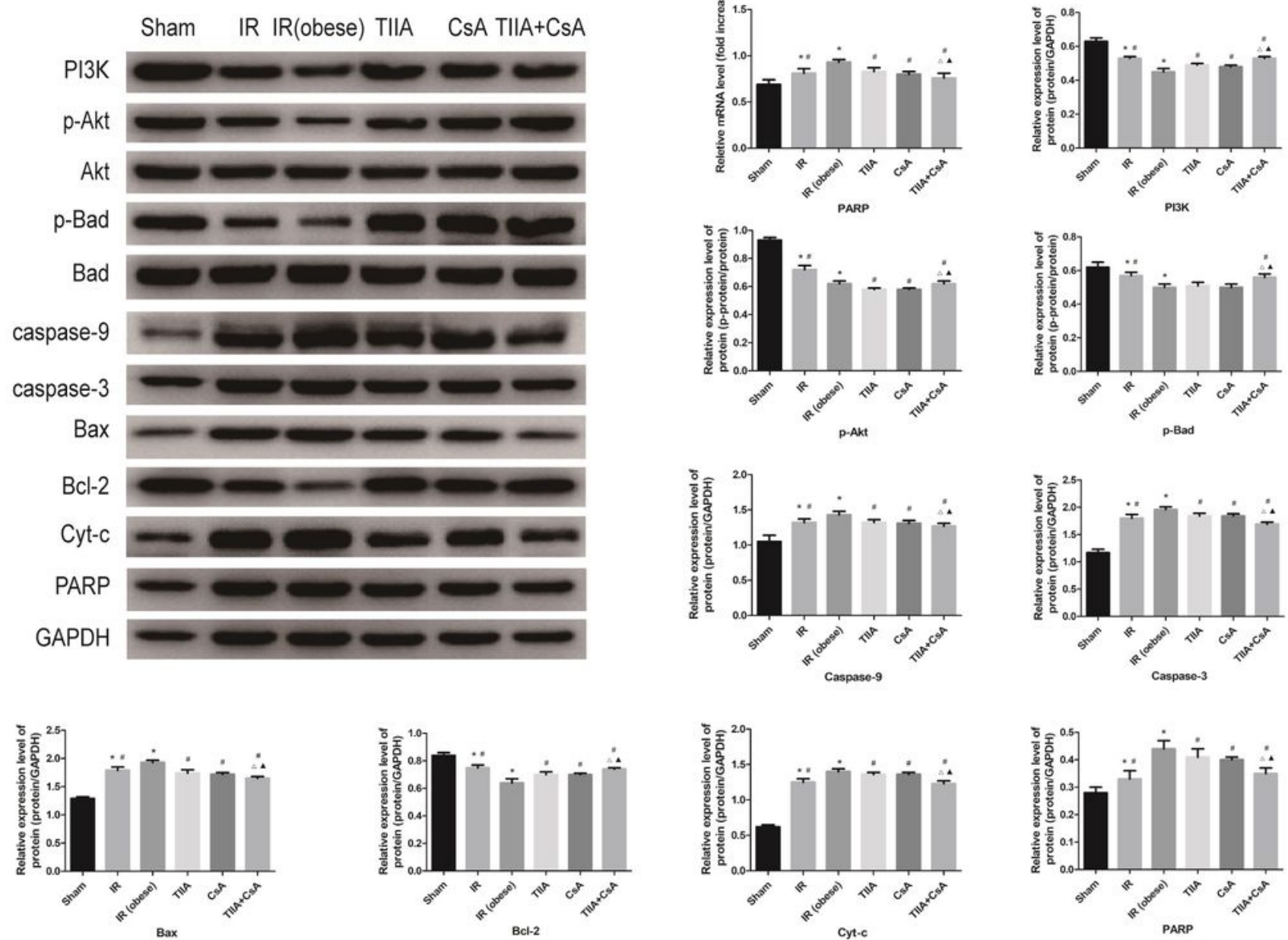

\section{Figure 6}

Tanshinone IIA (TIIA)+ cyclosporine A (CsA) modulated myocardial PI3K/Akt/Bad pathway The expression of PI3K, p-Akt, Akt, p-Bad, Bad, Bax, Bcl-2, Cyt-c, caspase-9, caspase-3, and PARP in mRNA (A) and protein (B) level. ${ }^{*} p<0.05$ versus sham group, $\# p<0.05$ versus IR (obese) group, $\triangle p<0.05$ versus TIIA group, $\Delta \mathrm{p}<0.05$ versus CsA group. 